\title{
Detection of period variations of eclipsing binaries in the Catalina Sky Survey
}

\author{
Athanasios Papageorgiou ${ }^{(0)}$, ${ }^{1,2}$ Márcio Catelan ${ }^{\circledR}$, 1,2 Panagiota-Eleftheria Christopoulou, ${ }^{3 \star}$ Andrew \\ J. Drake 4 and S. G. Djorgovski ${ }^{4}$ \\ ${ }^{1}$ Pontificia Universidad Católica de Chile, Facultad de Física, Instituto de Astrofísica, Av. Vicuña Mackenna 4860, 7820436 Macul, Santiago, Chile \\ ${ }^{2}$ Millennium Institute of Astrophysics, 7820436 Macul, Santiago, Chile \\ ${ }^{3}$ Department of Physics, University of Patras, 26500 Patra, Greece \\ ${ }^{4}$ California Institute of Technology, 1200 East California, Boulevard, CA 91225, USA
}

Accepted 2021 February 27. Received 2021 February 26; in original form 2020 July 30

\begin{abstract}
We present 126 eclipsing binary candidates among 4683 Catalina Sky Surveys (CSS) detached and semi-detached eclipsing binary systems (EBs) showing cyclic or quadratic period variations over a $12 \mathrm{yr}$ time span. By using inverse Gaussian profiles of the eclipses coupled with a Markov chain Monte Carlo procedure, times of minima (ToM) were calculated and diagrams with eclipse timing variations (ETVs) were constructed. Numerical tests were performed, involving synthetic EBs with period variations generated by the PHOEBE 2.0 engine and actual data for EBs with well-known period variations from the literature, to verify that the calculation of ToM variations for our CSS systems is reliable. A total of 63 out of the $126 \mathrm{EBs}$ show likely cyclic ETVs, while the remainder present quadratic behaviour instead. Periods, amplitudes, period change rates, and associated errors were determined by using sinusoidal and parabolic models. 12 out of the 63 EBs (19 per cent) that appear to exhibit periodic ETVs are low-mass candidates. Additionally, four out of 126 also have maximum quadrature light variations. The possibility that the cyclic variations are caused by the light traveltime effect due to the presence of a tertiary companion is investigated. The possible nature of the quadratic ETVs is also discussed.
\end{abstract}

Key words: methods: data analysis - surveys - (stars:) binaries (including multiple): close -(stars:) binaries: eclipsing.

\section{INTRODUCTION}

Eclipsing binary systems (EBs) are of crucial importance in stellar astrophysics, as they provide a means to measure properties of stars, including their masses, that can be difficult to measure otherwise. Yet, their orbital periods may change on time-scales of days or even decades, due to intrinsic, dynamical, or geometrical reasons. Intrinsic includes conservative or non-conservative (ejections and eruptions) mass transfer or angular momentum transfer between the components of the system (semidetached and contact configurations) but also magnetic torques as a result of the magnetic activity of one or both components (Applegate 1992; Lanza \& Rodonò 2004). The last two (dynamics and geometry) involve the presence of a third body around the system that perturbs its center of mass introducing a change in the distance between the binary components and the observer and gives rise to the light traveltime effect (LTTE). In the case of tight hierarchical triple stellar systems the short time-scale dynamical third body perturbations are analytically described by Borkovits et al. (2003, 2011, 2015). Thus, although one normally expects the eclipse events to be uniformly spaced in time, these phenomena will change the time interval between eclipses as observed from Earth in the case of LTTE or the actual period in the case of intrinsic or dynamical effects. In addition, apsidal motion, the change in the projected orbit

^E-mail: apapageo@astro.puc.cl (AP); pechris@physics.upatras.gr (PEC) along our line of sight associated with eccentric orbits, may also induce apparent period changes. Other physical phenomena that contribute to eclipse timing variations (ETVs) include star spots, spin-orbit transfer of angular momentum, barycentric, and asymmetric transverse velocities, the latter having recently been studied in detail by Conroy et al. (2018). The presence of a third body revolving around the EBs not only may induce ETVs but may play an important role in the formation and evolution of the EBs through the Kozai cycles with tidal friction mechanism (Eggleton \& Kisseleva-Eggleton 2006). Moreover, correlations between the maximum quadratic light variation (Papageorgiou et al. 2018) and possible ETVs will give strong evidence for the Applegate mechanism. Thus, the study of the ETVs history of EBs provides additional information to understand their evolution, orbital state, and probe their interior.

The residuals derived from the observed eclipse times, minus those expected from a simple assumed linear ephemeris, form an ETV diagram, known as 'Observed minus Computed' or ' $O-C$ ' diagram. The role of such diagrams in modern astrophysics can be reviewed in Sterken (2005) and further references therein. Many EBs have an even more complex ETV diagram in which all the above effects may be present. Therefore it is important to identify new candidate EBs with ETVs and investigate their origin.

The sample size of EBs with ETVs has increased significantly recently as researchers took advantage of the several sky survey projects that have catalogued EBs, based on their long-term photometry in different passbands. From Kepler data, Rappaport et al. (2013) found 
39 plausible candidates for triple-star systems. Conroy et al. (2014) identified 236 systems with a timing variation signal compatible with the presence of a third body whereas Borkovits et al. (2016) provided analytical models to 222 old and new ones. Lohr et al. (2015), analysing 13927 SuperWASP EBs, found 2 percent of the systems exhibited cyclic variations. Li et al. (2018) reported 91 EBs from the Microlensing Observations in Astrophysics (MOA; Sumi et al. 2013) EB catalogue with detected LTTE signals, suggesting the presence of tertiary companions with orbiting periods from $250 \mathrm{~d}$ to 28 yr. More recently, Hajdu et al. (2019) identified 992 potential hierarchical triple or multiple system candidates exhibiting LTTE by analysing a selection of 80000 EBs among 425193 variables of the Optical Gravitational Lensing Experiment (OGLE; Soszyński et al. 2016). Nevertheless, the selection effects, the different procedures (sampling, eclipsing timing methods, ETV analysis) and mainly the duration of the mission, set a strong bias for the detected periods or/and multiplicity rate of the detected candidate triple systems. For example, Kepler run for $4 \mathrm{yr}$ but data from MOA or OGLE-IV span two times longer ( $\sim 7$ and $9.5 \mathrm{yr}$, respectively).

Although a robust estimation of the frequency of EBs with period variations or/and tertiary companions needs a population synthesis that takes into account all the above selection effects and limitations, new long-term ground-based surveys are still valuable because they lengthen the data series and enrich the ETV diagrams of the already known EBs. Nevertheless they expand our sample with new candidate systems with ETVs and new period trends can be seen despite the individual uncertainties.

Given the interesting and special role of ETVs into the astrophysics of EBs, in this paper we are searching for period variation trends with the analysis of ETVs in EBs observed during the Catalina Sky Surveys (CSS) and specifically with eclipsing Algol (EA)-type like light curves. That means that the first goal of our study is not necessarily to detect LTTE signals in these systems that suggest the presence of tertiary companions but to reveal systems with noteworthy ETVs after evaluating the magnitude of variation. The second goal is to develop a new robust method to find candidate systems with ETVs from a long-term but not so dense populated ground based survey such CSS. In this way we want to identify within a large data set candidate binary systems with ETVs without the need to model analytically the binary system. This can open the door to preliminary identification of ETVs from ongoing ( Gaia; Gaia Collaboration 2018) or future very large multi-epoch surveys (Vera C. Rubin Observatory Legacy Survey of Space and Time, LSST; Ivezić et al. 2019) as well as to focus to more interesting systems with ETVs that deserve a more sophisticated analysis. In Section 2 we present the criteria for our sample selection and outline the steps of our method for the determination of times of minima (ToM) and the generation of the $O-C$ diagram. The results of the analysis of the ETVs are discussed in Section 3. Finally, our conclusions are given in Section 4.

\section{METHODS}

\subsection{Data selection}

In this study we focused on the EA-type binaries from CSS, and we use Catalina Surveys Data Release $2^{1}$ (CSDR2), augmented by additional, not publicly accessible data, spanning 12 yr (2004-2016). This sample includes a total of 4683 light curves (LCs); it was

${ }^{1}$ http://nesssi.cacr.caltech.edu/DataRelease/ described by Drake et al. (2014) and, more recently, in an updated catalogue by Papageorgiou et al. (2018). The new catalogue includes the physical classification of the EBs into detached/semidetached subclasses, using data spanning $12 \mathrm{yr}$. The sky coverage of the EBs is in the range of right ascension (RA) between 0 and $24 \mathrm{~h}$ and declination (Dec) between $-22^{\circ}$ and $+65^{\circ}$. The observations are taken unfiltered, and the magnitudes were transformed to an approximate $V$ magnitude ( $V_{\mathrm{CSS}}$, Drake et al. 2013). The photometry was performed by using the SEXTRACTOR aperture photometry program (Bertin \& Arnouts 1996). Graham et al. (2017) provided a correction factor to reduce the significantly overestimated original CSS photometric errors. Using the latter, Papageorgiou et al. (2019) extracted the physical parameters for $2281 \mathrm{EBs}$ from this sample by applying the Eclipsing Binaries via Artificial Intelligence (EBAI; Prša et al. 2008) Artificial Neural Network, and discussed the statistical properties of the physical parameter distributions.

For the purpose of this study we select 3026 EBs (out of 4683) based on the number of LC data points ( $N$ pts). Criteria like the quality of the LC, scatter, depth of minima has already been taken into account as the initial sample of 4683 LCs have been already been cleaned by using a sigma-clipping algorithm, period determined, and classified based on machine-learning techniques (Papageorgiou et al. 2018, 2019). The $\sim 90$ per cent of these EAs have periods $P$ $\leq 3 \mathrm{~d}$ (table 1; Papageorgiou et al. 2018). Furthermore the times were converted into Heliocentric Julian Date (HJD). Since some of the data sets do not contain enough data points as can be seen from fig. 1 of Papageorgiou et al. (2018) for a detailed examination only EBs with $N$ pts $\geq 400$ were finally accepted for further analysis. Furthermore Papageorgiou et al. (2019) found from EBAI analysis that the majority of these systems have eccentricity around 0 corresponding to circular orbits or eccentric orbits with argument of periastron $\omega$ $= \pm \pi / 2$.

\subsection{Times of minimum light computation}

Photometric surveys often rely on a limited number of observations per night, which makes it impossible to estimate the time of eclipses on a nightly basis. However, by suitably binning the time-series in larger LC time bins, each time of eclipse can be properly calculated. In our work, the time bins were selected on the basis of the number of points in each LC and the time difference between consecutive observations. Due to seasonal gaps in the observations, the time bins that were selected covered $\sim 300 \mathrm{~d}$, the exact timespan depending on the LC. The restriction of our research to cycles with periods longer that 300 days is a direct consequence of the frequency of the data points and the accuracy in the eclipse timing estimation. Therefore we know a priori that we cannot detect periodic variations in $O-C$ with periods shorter than $\sim 1$ yr. ETVs related with LTTE show that the majority of compact triples found in the stellar population having orbital periods well below 1 yr come from ultra-precise Kepler space mission, e.g in the study of Borkovits et al. (2016), 13 triples were reported (i.e. approximately 0.5 per cent 15 of the total number of the Kepler EBs) with outer period less than 150 d (Borkovits et al. 2015, 2016). Hajdu et al. (2017) identified five relatively short period Algol systems observed in the 100-150 d long CoRoT ETV data series for which the computed periods of the outer bodies are between 82 and 272 d. Although their solution does not satisfy the criterion that observations should cover at least two outer orbital periods (Conroy et al. 2014; Borkovits et al. 2016) their solutions were found to be physically consistent. Later short triple candidates were revealed from K2 data (Borkovits et al. 2019a, b) and most recently from TESS data (Borkovits et al. 2020). 

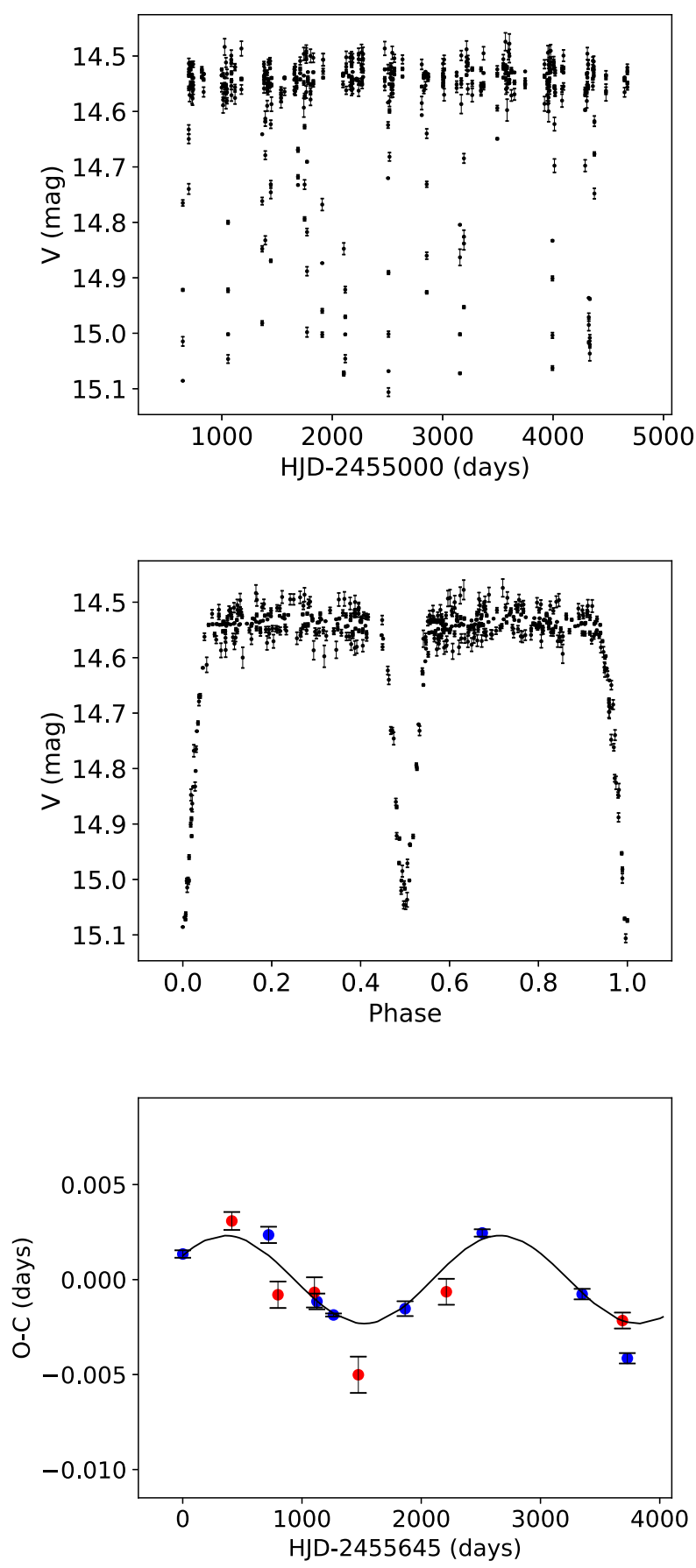

Figure 1. Test on a synthetic EB: raw (top panel) and phased (middle panel) synthetic LCs of a detached EB with period variations, generated using PHOEBE 2.0, are shown, along with the ETV diagram constructed with ToM calculated by our pipeline (bottom). Blue and red dots denote the ToM in the primary and secondary eclipses, respectively. The theoretical ETV curve is represented by the line.

Data points were phased separately in each time bin using the epoch and the period from Papageorgiou et al. (2018) and those out of the eclipses were removed before the fitting procedure by selecting a phase width of 0.2 . These boundaries were determined from the preliminary investigation of Papageorgiou et al. (2019) who found that the mean full width at half-maximum of the eclipse region of the LCs of the detached systems that constitute the majority of this sample of EBs with different ingress and egress is typically 0.060.07 of phase. Nevertheless in special cases if we want to fit a more complex profile to find the minima, the curvature of the LC outside the minima region has to be taken into account. The number of points in the resulting sub-samples were examined and at the last preparation stage before the fitting sub-samples with $N$ pts $\leq 10$ were rejected from the fitting phase.

Several works propose different methods of measuring ToM for automatic LC processing. The most used is the Kwee \& van Woerden method (Kwee \& van Woerden 1956) that is not suitable when the minimum is covered poorly. There are also methods that represent binary eclipses by simple quadratic or quartic functions near minimum (Rappaport et al. 2013; Borkovits et al. 2016) and methods that generate eclipse templates such as the semi automatic fitting procedure (AFP; Zasche et al. 2014), the template function as proposed by Mikulášek (2015) or any high order polynomial fit as used by Conroy et al. (2014) and the MAVKA software for approximating moments of extrema (both maxima and minima; Andrych et al. 2020). Here we present an automated procedure to calculate the times of eclipse minima by constructing and fitting a Gaussian function template to both primary and secondary eclipses in the following form:

$f(x)=C+d_{i} \times \exp \left[-\frac{\left(x-m_{i}\right)^{2}}{2 s_{i}^{2}}\right]$,

where $C$ is a constant, $s_{i}$ the half-width, $d_{i}$ the depth, and $m_{i}$ the phase shift, with $i=1,2$ for the primary and secondary eclipses, respectively. The use of Gaussians has been applied in the past by Hoffman et al. (2006) in the study of $O-C$ of selected Algol systems but also recently by Mowlavi et al. (2017) to OGLE-III LCs in LMC and Papageorgiou et al. (2018) to CRTS data to cover the need of an automated and robust LC processing. This method represents very well the eclipses except in the case of the contact systems with total eclipses part, which is not the case here.

The first step is to derive a mean LC from all seasons by binning the folded LC and consider only the portion belonging to primary or secondary eclipses. To avoid any distortion of the LC from seasonal variations due to any physical phenomena (e.g LTTE), we construct the median profile for each eclipse by using the median flux values in each 0.01 phase bin. Then we fitted each eclipse separately with equation (1) by adjusting its parameters. The best-fitting parameter search was performed using the method of Nelder-Mead Downhill Simplex, implemented in the LMFIT ${ }^{2}$ (Newville et al. 2016) PYTHON package. Equipped with these templates, we derive the individual ToM by fitting them to the phased LCs profile of the selected time bins ( 300 d). The profile was fitted via a Markov chain Monte Carlo (MCMC) procedure in which only the horizontal $\left(m_{i}\right)$ and the vertical $(C)$ shifts were adjusted, the other parameters remained fixed according to their pre-defined values. Thus, the phase shift for the certain epoch and the error were calculated. The epoch for the calculation of ToM by using the phase offset from the template was chosen as the deepest point of the eclipse as we consider that this is closest to the real time of observations. In this way it is possible that the epoch of the derived secondary ToM to be different from the primary. This procedure was performed using the affine invariant MCMC Ensemble sampler implemented in the EMCEE (ForemanMackey et al. 2013) Python package, using 25000 steps and 10 walkers for each sub-sample of the EB light curve, while the burn-in period was set to 5000 steps.

\footnotetext{
${ }^{2}$ http://dx.doi.org/10.5281/zenodo.11813
} 


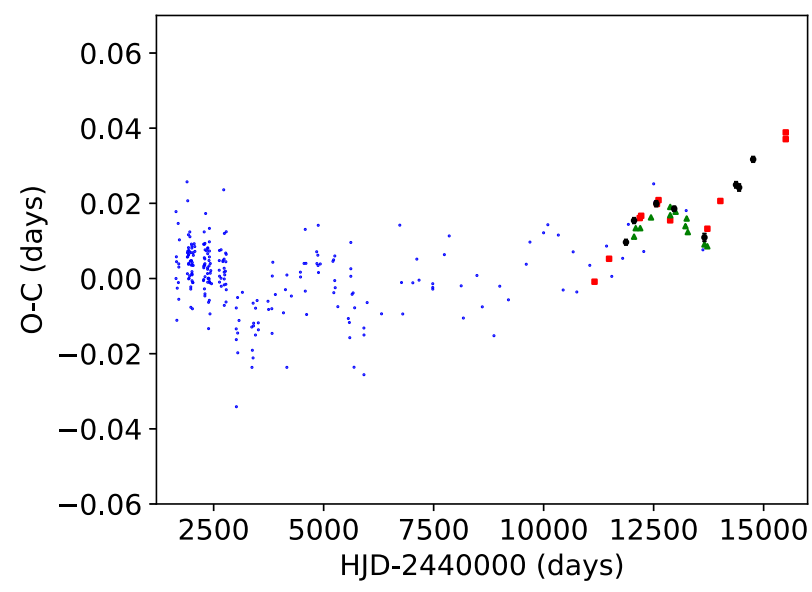

Figure 2. Test on the EB system VY Ceti: ETVs calculated from the ASAS LC using our pipeline (black circles) along with the minima calculated by Pilecki et al. (2007; green triangles) and other available minima from the literature (visual data: small blue dots; CCD observations: red squares).

We also performed, as a separate piece of the analysis a consistency test of the estimated minima with the AFP method. The result of the comparison (not presented here) shows that they do not alter the general picture of the $O-C$ variation as they verified that the derived amplitude and period modulation agree within the given errors with our results.

\subsection{Reliability of the method}

Two tests were run to check that period variations can be determined using the method described in Section 2.2, the first one on a synthetic LC and the second on a well-known EB with period variations.

\subsubsection{Test on a synthetic EB}

A synthetic EB LC for a detached system with a third component was generated using the PHOEBE 2.0 engine (Prša et al. 2016). In order to mimic the LC of an EB with a third component, a time-dependent perturbation was added to the times of eclipses. The synthetic data points were randomly selected and synthetic random noise was added in order to mimic the CSS data (Fig. 1, top panel). Furthermore, the times were selected from the observed time-series in order to mimic the CSS cadence (Fig. 1, middle panel). The primary and secondary times of eclipses of the synthetic EB calculated (Fig. 1, bottom panel) with the template fitting described in Section 2.2 (blue and red circles, respectively) are in good agreement with the theoretical ETVs (solid black line).

\subsubsection{Test on a real EB}

As we did not find any known EB with a well-known cyclic ETV that is well covered from CRTS and fulfills all the above selection criteria of the method, the pipeline was tested in the real world using the EB system VY Cet, with a well-known cyclic ETV. The ToM were calculated using the LC from the All Sky Automated Survey (ASAS, Pojmanski 1997; Pojmanski, Pilecki \& Szczygiel 2005) and template fitting. Fig. 2 shows that the ETVs calculated from the ASAS LC using our pipeline (black dots) are in excellent agreement with the minima calculated by Pilecki, Fabrycky \& Poleski (2007) and other available minima from the literature.

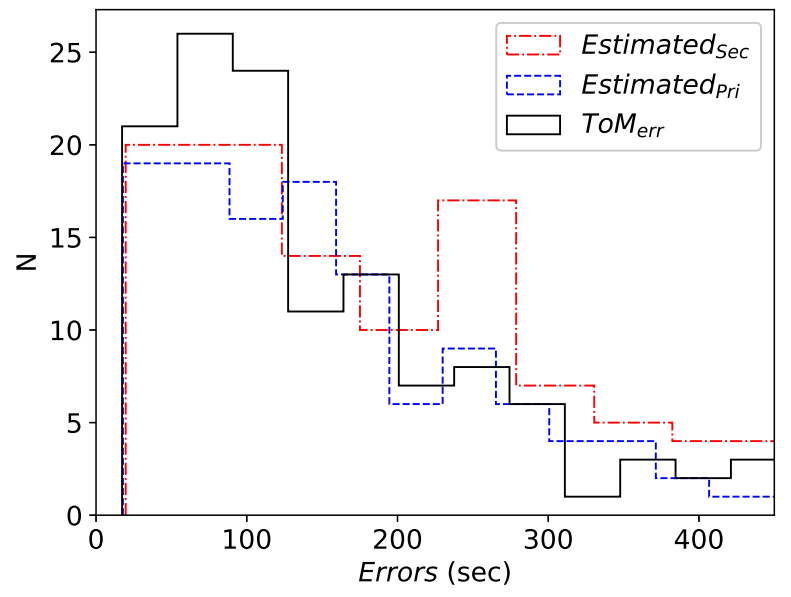

Figure 3. Comparison between the estimated ToM uncertainties based on the equation (2) and the LC properties of $126 \mathrm{CSS}$ EBs for the primary (dashed blue line) and secondary minimum (dashed-dotted red line), and the calculated ToM uncertainties from the MCMC procedure (black solid line).

\subsection{Analysis of the ETVs}

The minimum uncertainty $(\Delta t)$ of the calculated ToM depends on the number $N$ of observed points during the eclipses, its depth $d$, the duration of the minimum $D$, and the photometric errors $\sigma$ of the LC, according to the following expression (Pribulla et al. 2012):

$\Delta t=\frac{D \sigma}{2 d \sqrt{N}}$

To compare the performance of the MCMC procedure in the ToM error calculation, the uncertainties were estimated from the LC phenomenological parameters given by Papageorgiou et al. (2018) and equation (2) for the primary and secondary eclipses. As shown in Fig. 3, the estimated uncertainties are in very good agreement. Additionally, erroneous minima calculated from a few points in the eclipse, or in shallow eclipses, were discarded by using the modified z-score method (Iglewicz \& Hoaglin 1993). Thereafter, only EBs with calculated ToM $\geq 6$ were accepted for the $O-C$ analysis. This selection resulted in 2604 EBs.

Using the epoch, the initial period from Papageorgiou et al. (2018), and the new ToM described in Section 2.2, a linear ephemeris was calculated for each EB for the primary minima in the form of

$\operatorname{HJD}_{c}(E)=\mathrm{HJD}(0)+P \times E$,

where $\operatorname{HJD}_{c}(E)$ denotes the time of $E$ th eclipse, $\operatorname{HJD}(0)$ the reference minimum, and $P$ the mean orbital period of the binary (Table 1). Then, the $O-C$ diagram of each $\mathrm{EB}$ was constructed according to the following equation:

$O-C=\operatorname{HJD}_{O}(E)-\operatorname{HJD}_{C}(E)$,

where $\operatorname{HJD}_{O}(E)$ and $\operatorname{HJD}_{C}(E)$ are the observed and the calculated ToM, respectively. After this, the $2604 O-C$ diagrams were fitted with a sinusoidal or/and a parabolic function using the LevenbergMarquardt algorithm (Levenberg 1944; Marquardt 1963) adjusting also the ephemeris. To avoid over-fitting, each EB was assigned a label according to its Bayesian Information Criterion (BIC; Schwarz 1978). To choose the most appropriate model, we demand a BIC difference $(\triangle \mathrm{BIC})$ of 5 from the null hypothesis (no period change). This reduced the number of investigated EBs to 577. Moreover, all the $O-C$ diagrams were visually inspected. Sinusoidal variations with mean ToM error-to-amplitude ratio greater than 1.5 or parabolic 
Table 1. Linear ephemeris (epoch HJD and period $P_{\text {bin }}$ ) for 126 CSS EBs and mean error of the calculated ToM. Symbols '-' and ' + ' denote the lower and upper bounds, respectively, of the $1 \sigma$ error derived from the Monte Carlo (MC) parameter distributions. (The full table can be obtained in machine readable form in the electronic edition of the paper).

\begin{tabular}{|c|c|c|c|c|c|c|c|}
\hline Name & $\begin{array}{l}\mathrm{RA}_{\mathrm{J} 2000} \\
\text { (h:m:s) }\end{array}$ & $\begin{array}{l}\operatorname{Dec}_{J 2000} \\
\left(\vdots^{\prime}:^{\prime \prime}\right)\end{array}$ & $\begin{array}{c}\text { HJD }-2400000 \\
\text { (d) }\end{array}$ & $\begin{array}{c}\text { HJD }_{\text {err }} \\
(\mathrm{d}[-,+])\end{array}$ & $\begin{array}{r}P_{\text {bin }} \\
(d)\end{array}$ & $\begin{array}{c}\left(P_{\text {bin }}\right)_{\text {err }} \\
\left(\mathrm{d} \times 10^{-7}[-,+]\right)\end{array}$ & $\begin{array}{l}\langle\text { ToM }\rangle_{\text {err }} \\
\text { (d) }\end{array}$ \\
\hline CSS_J002011.4 + 181918 & $00: 20: 11.40$ & 18:19:18.70 & 57282.75397 & $0.00078,0.00084$ & 1.1390399 & $4.4,4.1$ & 0.0021 \\
\hline CSS_J003703.4 + 213021 & 00:37:03.41 & 21:30:21.30 & 57363.74899 & $0.00244,0.00284$ & 2.0818985 & $23.1,23.9$ & 0.0033 \\
\hline CSS_J010700.6 + 251138 & 01:07:00.62 & $25: 11: 38.40$ & 57386.50607 & $0.00199,0.00188$ & 2.2838341 & $15.9,15.4$ & 0.0024 \\
\hline CSS_J011119.5 + 331912 & 01:11:19.52 & $33: 19: 12.50$ & 57388.22034 & $0.00101,0.00091$ & 0.8421917 & $3.7,3.5$ & 0.0019 \\
\hline CSS_J022053.1 + 165300 & 02:20:53.11 & $16: 53: 00.70$ & 57283.91750 & $0.00062,0.00066$ & 0.4649798 & $1.4,1.4$ & 0.0011 \\
\hline CSS_J023303.0 + 105943 & 02:33:03.01 & $10: 59: 43.70$ & 57408.48849 & $0.00021,0.00020$ & 0.4203023 & $0.5,0.5$ & 0.0005 \\
\hline
\end{tabular}

variations with period changes greater than $10^{-10} \mathrm{~d}$ per cycle were finally accepted as EBs with period changes. Since there is always the problem of how many data points one may need to be sure of a meaningful result, in order to confirm the detected ETVs we applied the process again by fitting each LC 100 times, excluding randomly 10 percent of the total $O-C$ points in each fit. EBs for which the linear model was preferable in more than 10 fits were then excluded. Fig. 4 shows representative examples of the $O-C$ diagram analysis and fitting procedure.

\section{RESULTS}

In total we analysed 2604 eclipsing Algol-type binaries from the CSS survey. By applying the method and the criteria described in Section 2.4, we arrived at the final sample of 126 EBs with significant period changes which are listed in Table 1 . This table provides the CSS ID, the right ascension $\left(\mathrm{RA}_{\mathrm{J} 2000}\right)$ and declination $\left(\operatorname{Dec}_{\mathrm{J} 2000}\right)$, the linear ephemeris (the epoch HJD and the period $P_{\text {bin }}$ ) with the MC-derived errors and the mean error of the calculated ToM. From this sample, we identified $63 \mathrm{EBs}$ with cyclic variations and 63 EBs with more likely quadratic behaviour. These are presented in Tables 2 and 3, respectively, along with their derived parameters and the corresponding uncertainties. Furthermore, in order to achieve a better estimation of the parameter errors, $\mathrm{MC}$ simulations were performed. Thus, synthetic $O-C$ diagrams were created from the original ToM by adding random displacements to the ToM within their errors, the latter being randomly sampled from a Gaussian distribution with zero mean and standard deviation corresponding to the ToM error. We performed this procedure by fitting three models (linear, parabolic, sinusoidal) 1000 times for each individual EB. The parameter errors were finally calculated using the 16th and 84th quantile of the resulted distributions for the lower and upper error, respectively.

Table 2 provides the CSS ID, the amplitude ( $A m p_{\text {mod }}$ ) of the cyclic variation, the period modulation $\left(\right.$ Per $\left._{\text {mod }}\right)$, the MC-based parameter errors, and the BIC criterion $(\triangle \mathrm{BIC})$. Table 3 , in turn, provides the CSS ID, the period change rate $\left(\frac{d P_{\text {bin }}}{d E}\right)$, the MC-based errors, and the BIC criterion $(\triangle \mathrm{BIC})$. The ETV plots for the total sample of 126 EBs are presented in Appendix A (Figs A1-A10).

Fig. 5 shows the distribution of the derived parameters for the sample of 126 CSS candidates with period variation. We have to note that the detected periods are biased due to the observing time span $(\sim 12 \mathrm{yr})$ and the time-binned subsets $(\sim 1 \mathrm{yr})$, whereas the detected amplitudes have a lower limit due to the accuracy of our analysis $(\sim 2 \mathrm{~min})$.

\subsection{LTTE analysis}

According to Table 2, there are 63 systems with a cyclic change in orbital period. Assuming that the period modulation is caused by a third companion orbiting the binary system, the lower limit of the mass of the third body can be calculated from the mass function $f\left(M_{3}\right)$, defined as

$f\left(M_{3}\right)=\frac{4 \pi^{2} \alpha_{12}^{3} \sin ^{3} i_{3}}{G P_{3}^{2}}=\frac{M_{3}^{3} \sin ^{3} i_{3}}{\left(M_{12}+M_{3}\right)^{2}}$,

where $M_{3}, i_{3}$, and $P_{3}$ are the mass, inclination, and period of the tertiary object, respectively; $M_{12}$ is the total mass of the binary; and $\alpha_{12}$ is the semi-major axis of the binary orbit. The latter is connected with the LTTE amplitude (Irwin 1959) by

$A_{\mathrm{LTTE}}=\frac{\alpha_{12} \sin i_{3}}{c} \sqrt{1-e^{2} \cos \omega_{3}}$,

where $e_{3}$ is the eccentricity, $\omega_{3}$ is the argument of periastron of the orbit of the third object around the EB's centre of mass, and $c$ is the speed of light. Since the model used for the ETVs fitting is a simple sinusoidal curve due to the limited number of points in our ETVs diagram, zero eccentricity of the third body was assumed $\left(e_{3}=0\right)$. New photometric observations or computation of ToM from other surveys will give the opportunity to apply the analytical formula of Irwin (1959) that takes into account the eccentricity of the potential third body.

To evaluate equations (5) and (6), we take values for the period modulation $P_{3}=P e r_{\text {mod }}$ and the amplitude $A_{\mathrm{LTTE}}=A m p_{\text {mod }}$ from Table 2, assuming $i_{3}=90^{\circ}$ and $M_{12} \sim 2 \mathrm{M}_{\odot}$. In Fig. 6 we illustrate how the predicted possible minimum masses of third bodies $\left(M_{3}\right)$ correlate with their periods $\left(P_{3}\right)$ and LTTE amplitude. As shown in this figure, we can detect EBs with a small third object of mass no less than $0.1 \mathrm{M}_{\odot}$ with our pipeline. Thus, we cannot detect any circumbinary planetary-mass companion, but primarily stellar lowmass tertiary companions. In particular, the vast majority of the candidate triple systems have $M_{3}<0.6 \mathrm{M}_{\odot}$ and LTTE amplitude between 5 and $10 \mathrm{~min}$. For five or our binaries the minimum predicted mass of the potential third body is comparable with the assumed total mass of the system, although their errors are the largest. Unfortunately, only one, CSS_J075017.2 + 400441 has been studied and best explained as metal poor main sequence pair of $\sim(1.2+0.8) \mathrm{M}_{\odot}$ stars (Brown, Kilic \& Gianninas 2017). For the 63 triple candidates, we also plot the binary period versus the period of the third object in Fig. 7. This plot indicates a roughly flat distribution with period ratios $\left(P_{3} / P_{\text {bin }}\right)$ between $10^{3}$ and $10^{5}$, in accordance with the 992 OGLE-IV potential triple candidates (Hajdu et al. 2019) that are also shown for comparison in the same figure. 
CSS $1030325.0+250550$
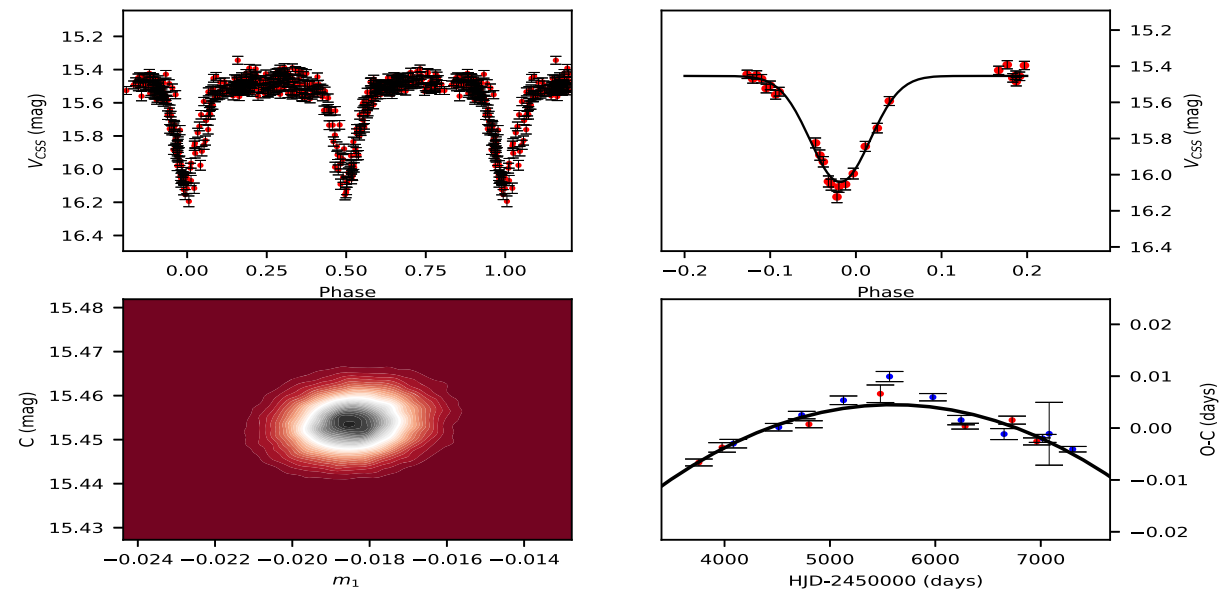

CSS J142938.8+054621
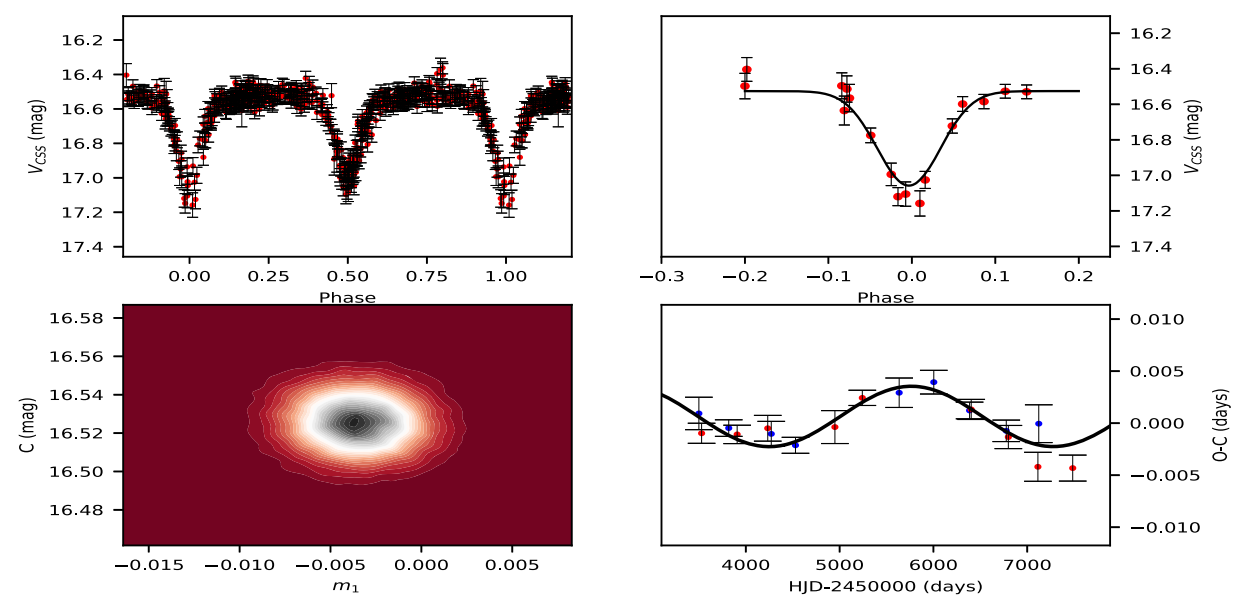

CSS $1081715.9+130132$
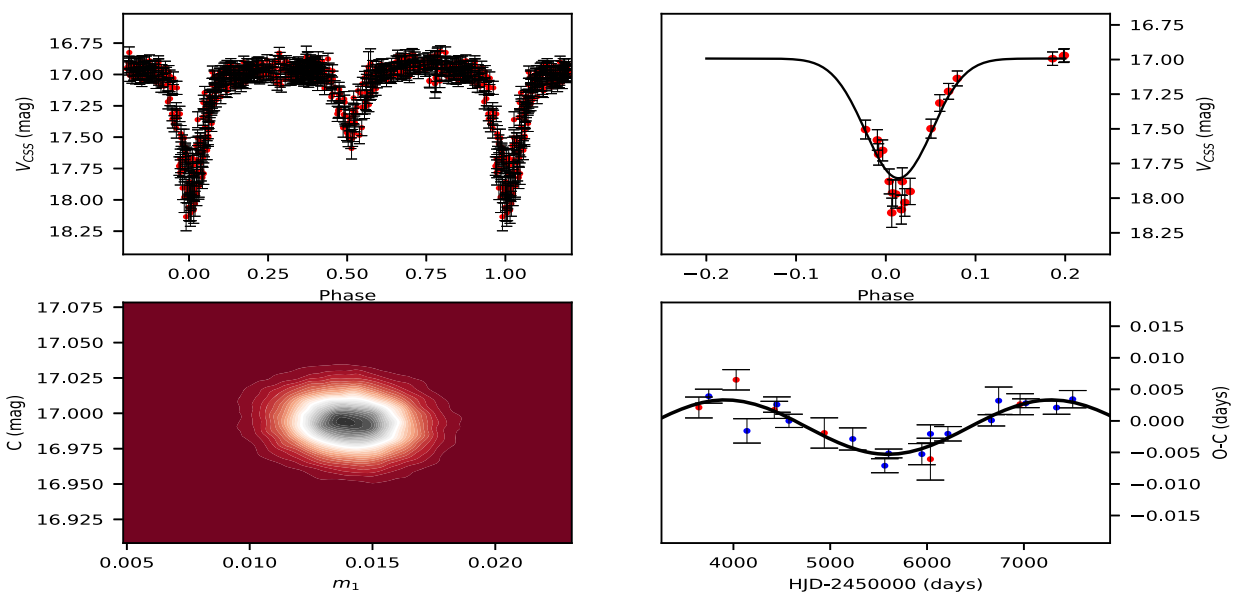

Figure 4. Representative examples of EBs with period variations found in CSS data. Three stars are shown, namely (from top to bottom) CSS_J030325.0 + 250550, CSS_J142938.8 + 054621, and CSS_J081715.9 + 130132. For each star, four panels are displayed, as follows: folded light curve (upper left), ETV data together with the sinusoidal or parabolic model fit (lower right), ToM fit with a Gaussian function (upper right), and MCMC procedure in which only the horizontal and vertical shifts ( $m$ and $C$, respectively) were adjusted (lower left). 
Table 2. Amplitudes ( $\left.A m p_{\text {mod }}\right)$ and periods $\left(P_{\text {mod }}\right)$ for 63 CSS EBs with cyclic ETV variation. Symbols '-' and ' + ' denote the lower and upper bounds, respectively, of the $1 \sigma$ parameter error derived from the MC parameter distributions.

\begin{tabular}{|c|c|c|c|c|c|}
\hline Name & $\begin{array}{l}A m p_{\text {mod }} \\
\text { (d) }\end{array}$ & $\begin{array}{c}\left(A m p_{\text {mod }}\right)_{\text {err }} \\
(\mathrm{d}[-,+])\end{array}$ & $\begin{array}{c}P e r_{\text {mod }} \\
(\mathrm{yr})\end{array}$ & $\begin{array}{c}\left(\operatorname{Per}_{\mathrm{mod}}\right)_{\mathrm{err}} \\
(\mathrm{yr}[-,+])\end{array}$ & $\triangle \mathrm{BIC}$ \\
\hline CSS_J011119.5 + 331912 & 0.0071 & $0.0007,0.0008$ & 7.07 & $0.25,0.26$ & 19.90 \\
\hline CSS_J020441.1 + 221937 & 0.0015 & $0.0004,0.0003$ & 8.57 & $0.59,0.51$ & 10.58 \\
\hline CSS_J022053.1 + 165300 & 0.0022 & $0.0006,0.0006$ & 7.22 & $0.63,0.62$ & 6.75 \\
\hline CSS_J031340. $8+311303$ & 0.0066 & $0.0013,0.0014$ & 7.96 & $0.44,0.49$ & 15.00 \\
\hline CSS_J031452.7 + 313454 & 0.0035 & $0.0007,0.0006$ & 8.77 & $0.76,0.93$ & 18.08 \\
\hline CSS_J031556.8 + 313849 & 0.0018 & $0.0004,0.0004$ & 5.53 & $0.29,0.27$ & 14.15 \\
\hline CSS_J032325.8 + 010634 & 0.0013 & $0.0002,0.0002$ & 7.74 & $0.65,0.47$ & 14.11 \\
\hline CSS_J033117.7 + 004210 & 0.0049 & $0.0003,0.0004$ & 7.93 & $0.16,0.17$ & 14.31 \\
\hline CSS_J033531.2 + 355325 & 0.0082 & $0.0011,0.0011$ & 9.47 & $0.68,0.72$ & 13.50 \\
\hline CSS_J034800.2 + 285200 & 0.0067 & $0.0009,0.0011$ & 7.44 & $0.43,0.55$ & 8.49 \\
\hline CSS_J040632.6+ 011333 & 0.0057 & $0.0005,0.0005$ & 6.60 & $0.32,0.27$ & 11.38 \\
\hline CSS_J041441.5 + 071434 & 0.0101 & $0.0032,0.0032$ & 8.01 & $0.36,0.56$ & 10.64 \\
\hline CSS_J042420.5 + 293049 & 0.0078 & $0.0012,0.0012$ & 6.79 & $0.24,0.24$ & 5.87 \\
\hline CSS_J044339.0 + 230903 & 0.0143 & $0.0017,0.0017$ & 5.16 & $0.14,0.18$ & 11.34 \\
\hline CSS_J045817.6 + 113440 & 0.0080 & $0.0005,0.0005$ & 7.07 & $0.23,0.28$ & 11.04 \\
\hline CSS_J050059.2 + 085800 & 0.0067 & $0.0011,0.0013$ & 6.61 & $0.41,0.66$ & 7.21 \\
\hline CSS_J050856.0 + 074348 & 0.0065 & $0.0006,0.0006$ & 9.28 & $0.33,0.36$ & 27.32 \\
\hline CSS_J051407.3 + 010227 & 0.0016 & $0.0002,0.0002$ & 7.56 & $0.46,0.50$ & 22.39 \\
\hline CSS_J071249.1 + 405512 & 0.0076 & $0.0011,0.0012$ & 5.51 & $0.36,0.40$ & 5.08 \\
\hline CSS_J072551.2 + 390140 & 0.0049 & $0.0007,0.0008$ & 6.57 & $0.20,0.21$ & 14.49 \\
\hline CSS_J074431.3 + 240414 & 0.0053 & $0.0007,0.0007$ & 5.38 & $0.22,0.28$ & 10.13 \\
\hline CSS_J074528.3 + 372601 & 0.0059 & $0.0005,0.0005$ & 6.88 & $0.16,0.19$ & 17.25 \\
\hline CSS_J075017.2 + 400441 & 0.0177 & $0.0045,0.0050$ & 7.66 & $0.36,0.43$ & 40.46 \\
\hline CSS_J080150.8 + 343755 & 0.0194 & $0.0043,0.0045$ & 7.62 & $0.55,0.39$ & 10.91 \\
\hline CSS_J080205.8 + 433228 & 0.0068 & $0.0020,0.0023$ & 7.19 & $0.48,0.63$ & 6.71 \\
\hline CSS_J080324.8+ 195206 & 0.0025 & $0.0006,0.0005$ & 5.69 & $0.89,0.64$ & 8.94 \\
\hline CSS_J081715.9 + 130132 & 0.0043 & $0.0004,0.0003$ & 9.22 & $0.58,0.76$ & 39.20 \\
\hline CSS_J081947.2 + 023144 & 0.0128 & $0.0034,0.0026$ & 4.94 & $0.15,0.27$ & 11.17 \\
\hline CSS_J082525.1 + 015220 & 0.0110 & $0.0025,0.0026$ & 8.81 & $0.86,0.98$ & 10.77 \\
\hline CSS_J083636.3 + 472007 & 0.0045 & $0.0017,0.0018$ & 6.36 & $0.52,0.61$ & 15.55 \\
\hline CSS_J083745.3 + 022122 & 0.0044 & $0.0002,0.0002$ & 8.06 & $0.13,0.14$ & 16.97 \\
\hline CSS_J084458.7 + 051736 & 0.0039 & $0.0010,0.0011$ & 6.07 & $0.50,0.26$ & 5.63 \\
\hline CSS_J084911.7 + 112816 & 0.0018 & $0.0004,0.0004$ & 7.21 & $0.60,0.66$ & 9.66 \\
\hline CSS_J090846.5 + 444716 & 0.0016 & $0.0003,0.0002$ & 6.23 & $0.28,0.27$ & 21.00 \\
\hline CSS_J091534.0 + 333404 & 0.0017 & $0.0004,0.0005$ & 8.70 & $1.06,1.48$ & 11.45 \\
\hline CSS_J093348.6 + 083551 & 0.0048 & $0.0003,0.0003$ & 8.56 & $0.19,0.18$ & 12.55 \\
\hline CSS_J095457.0 + 322951 & 0.0038 & $0.0008,0.0008$ & 8.52 & $0.92,0.84$ & 18.28 \\
\hline CSS_J100900.3 + 170114 & 0.0052 & $0.0011,0.0012$ & 7.17 & $0.92,0.64$ & 6.09 \\
\hline CSS_J102723.5 + 271147 & 0.0086 & $0.0014,0.0015$ & 8.74 & $0.93,0.97$ & 26.00 \\
\hline CSS_J105105.7 + 224456 & 0.0076 & $0.0005,0.0005$ & 6.40 & $0.10,0.09$ & 12.55 \\
\hline CSS_J112729.0 + 020034 & 0.0035 & $0.0002,0.0003$ & 11.35 & $1.09,1.16$ & 23.38 \\
\hline CSS_J112751.6 + 244405 & 0.0023 & $0.0002,0.0002$ & 9.69 & $0.29,0.31$ & 10.62 \\
\hline CSS_J120619.5 + 315825 & 0.0038 & $0.0005,0.0006$ & 7.94 & $0.36,0.36$ & 10.25 \\
\hline CSS_J123622.4 + 343346 & 0.0016 & $0.0004,0.0005$ & 7.48 & $0.67,0.85$ & 16.14 \\
\hline CSS_J140415.6 + 003034 & 0.0032 & $0.0003,0.0004$ & 9.43 & $0.59,0.53$ & 12.38 \\
\hline CSS_J142910.4 + 112549 & 0.0043 & $0.0005,0.0006$ & 8.64 & $0.22,0.25$ & 16.35 \\
\hline CSS_J142938.8 + 054621 & 0.0029 & $0.0004,0.0004$ & 8.30 & $0.34,0.40$ & 20.28 \\
\hline CSS_J145327.8 + 005322 & 0.0022 & $0.0001,0.0001$ & 6.50 & $0.25,0.47$ & 14.52 \\
\hline CSS_J160357.9+ 211450 & 0.0042 & $0.0020,0.0017$ & 5.45 & $0.28,0.24$ & 18.84 \\
\hline CSS_J160810.2 + 291641 & 0.0061 & $0.0004,0.0004$ & 7.94 & $0.14,0.14$ & 15.61 \\
\hline CSS_J161641.1 + 161035 & 0.0043 & $0.0008,0.0009$ & 8.30 & $0.49,0.52$ & 11.10 \\
\hline CSS_J161829.2 + 284430 & 0.0180 & $0.0043,0.0049$ & 6.52 & $0.55,0.63$ & 17.79 \\
\hline CSS_J162156.8 + 153000 & 0.0019 & $0.0001,0.0002$ & 6.69 & $0.21,0.10$ & 13.50 \\
\hline CSS_J162421.8 + 102711 & 0.0031 & $0.0002,0.0002$ & 7.65 & $0.09,0.09$ & 11.74 \\
\hline CSS_J162604.2 + 091849 & 0.0023 & $0.0004,0.0004$ & 8.03 & $0.35,0.40$ & 8.90 \\
\hline CSS_J163008.7 + 144614 & 0.0022 & $0.0007,0.0006$ & 8.31 & $1.40,1.20$ & 6.47 \\
\hline CSS_J163252.0 + 293856 & 0.0100 & $0.0008,0.0008$ & 7.24 & $0.21,0.20$ & 23.05 \\
\hline CSS_J164643.0 + 212805 & 0.0062 & $0.0027,0.0033$ & 7.86 & $0.65,0.88$ & 11.02 \\
\hline CSS_J213637.4 + 042323 & 0.0048 & $0.0001,0.0001$ & 8.02 & $0.10,0.11$ & 12.99 \\
\hline CSS_J213638.8 + 031530 & 0.0073 & $0.0020,0.0022$ & 8.09 & $0.45,0.41$ & 10.63 \\
\hline CSS_J220219.9 + 073905 & 0.0036 & $0.0009,0.0093$ & 12.48 & $1.65,1.65$ & 6.32 \\
\hline
\end{tabular}


Table 2 - continued

\begin{tabular}{lccccr}
\hline Name & $\begin{array}{c}A m p_{\text {mod }} \\
(\mathrm{d})\end{array}$ & $\begin{array}{c}(\text { Amp } \text { mod })_{\text {err }} \\
(\mathrm{d}[-,+])\end{array}$ & $\begin{array}{c}P e r_{\text {mod }} \\
(\mathrm{yr})\end{array}$ & $\begin{array}{c}\left(\text { Per }_{\text {mod }}\right)_{\text {err }} \\
(\mathrm{yr}[-,+])\end{array}$ & $\Delta \mathrm{BIC}$ \\
\hline CSS_J221137.4+125934 & 0.0070 & $0.0015,0.0015$ & 4.08 & $0.18,0.18$ & 18.81 \\
CSS_J233313.7+283745 & 0.0027 & $0.0012,0.0007$ & 7.46 & $0.43,0.44$ & 8.88 \\
\hline
\end{tabular}

An alternative cause of sinusoidal ETVs is provided by the Applegate mechanism (Applegate 1992). The latter explains orbital period variations as a result of stellar magnetic activity due to changes in the stellar quadrupole moment. For this sample, we computed the period change relative to the binary period $\left(\frac{\Delta P_{\text {bin }}}{P_{\text {bin }}}\right)$ via

$\frac{\Delta P_{\text {bin }}}{P_{\text {bin }}}=\frac{4 \pi \times A m p_{\text {mod }}}{P e r_{\text {mod }}}$,

using the amplitude $A m p_{\text {mod }}$ and the modulation period $P e r_{\text {mod }}$ as displayed in Table 2 . The resulting relative period change $\frac{\Delta P_{\text {bin }}}{P_{\text {bin }}}$, of the order of $10^{-6}-10^{-7}$, agree with previously reported ones (Wolf et al. 2016; Zhang et al. 2018; Bin et al. 2019). Unfortunately, since we do not know the absolute parameters of any particular binary system, we are not in a position to properly estimate the required energy as a fraction of the available energy in the magnetically active star that would render the Applegate mechanism feasible for our systems. Otherwise, such a test could be carried out by applying the models proposed by Völschow et al. (2016) or Navarrete et al. (2018). Nevertheless, we have to stress that one of the Applegate mechanism's testable predictions is the correspondence of the luminosity variations in the active star with the modulations in the orbital period. Such maximum light variations were detected and discussed in Papageorgiou et al. (2018) for 119 systems. Among the latter, however, there are only four EBs that exhibit ETVs (one sinusoidal and three parabolic). The cause of this different result is the criteria we have imposed in every step of our method of ETV detection (Section 2.4). Only 20 out of these 119 EBs passed both the $\mathrm{BIC}$ criteria and the criterion based on the number of points per LC and finally only 4 out of the 20 EBs passed the criterion of mean ToM error-to-amplitude ratio. Another explanation to this result is that the maximum quadratic light variation due to the Applegate mechanism is not so efficient. In conclusion, we also have to note that another possible explanation for the observed period changes could be the slow movement of starspots on the surface of the binary components, since the starspot activity cycle shares with the Applegate mechanism the same period of the magnetic cycle of the magnetically active star. The time displacement of the observed ToM, due to spots, is typically $\sim 1-5 \times 10^{-3} \mathrm{~d}$ from the true ToM (Pribulla et al. 2000).

\subsection{Systems with linear period change trend}

Table 3 contains 63 systems that display ETVs with an upward or downward parabolic shape, indicating a linear change in the period of the order of $\sim 10^{-6}-10^{-7} \mathrm{~d} \mathrm{yr}^{-1}$. Additionally, to test the derived values of the period change rate, a harmonic fit to the sample of 63 LCs with parabolic ETVs was performed, based on the model described in Pilecki et al. (2007), as follows:

$$
\begin{aligned}
& X(t)=\sum_{m=0}^{H} A_{m} \cos (m \theta(m, t)), \\
& \theta(m, t)=\omega\left(t-T_{0, m}\right)+\frac{d \omega}{d t}\left(\frac{t^{2}}{2}-\frac{T^{2}}{12}\right),
\end{aligned}
$$

where $T$ is the time interval between the first and the last photometric observation of each EB, $H$ the number of harmonics, $\omega$ the angular frequency of the binary, and $T_{0, m}$ the phase on the order of harmonic $m$. The observation times $t$ are relative to the centre of the observed time interval. The amplitudes were initialized by fitting the model in phase domain. All parameters were then adjusted by fitting the model in the time domain, with the exception of $\frac{d \omega}{d t}$, which was held fixed at zero initially. Finally, $\frac{d \omega}{d t}$ and $\omega$ were also adjusted, in order to converge to the final solution. For this fitting procedure, the Levenberg-Marquardt (Levenberg 1944; Marquardt 1963) and Nelder-Mead Downhill Simplex (Nelder \& Mead 1965) algorithms were incorporated through the LMFIT PYTHON package. A total of 58 out of 63 systems with parabolic ETVs were successfully fitted. We have verified that, as long as $H \geq 10$ (as required in order to provide a good representation of Algol-type LCs), the results are in agreement with the findings from the ETVs fitting. Fig. 8 provides a comparison of the results from both methods.

To conclude, we emphasize that we cannot know a priori if the parabolic variation represents part of a sinusoidal variation, i.e. a potential LTTE signal with a period longer than the timespan of our data ( $\sim 12 \mathrm{yr})$. The largest period decrease was observed for the system CSS_J045118.8 +243511 , with a rate of $-2.34 \times 10^{-5} \mathrm{~d} \mathrm{yr}^{-1}$. If such a period decrease is due to magnetic braking of the secondary component, we can calculate the mass loss rate for given binary parameters. We do not analyse other possibilities of the $O-C$ variability, such as mass transfer, since the majority of the systems ( $\sim 2 / 3$ ), according to the classification of Papageorgiou et al. (2018), are detached systems. Nevertheless, we note that we do not exclude this as a possible mechanism for the potential semidetached systems ( $\sim 1 / 3$ of the sample), since in classical Algols mass transfer from the less massive component and mass loss via magnetic braking should be considered (Ibanoğlu et al. 2006; Erdem \& Öztürk 2014).

\section{CONCLUSIONS}

We exploited the entire sample of 4683 Algol-type EBs from CSS, searching for signs of period variations. Our proposed method to search for the latter revealed $126 \mathrm{~EB}$ systems that should be considered for follow-up observations and systematic study of the ETVs.

Out of the 63 systems in the latter sample that appear to exhibit periodic ETVs, 12 (or 19 per cent) are low-mass candidates (Papageorgiou et al. 2018) and have their initial parameters derived by EBAI or the template method Papageorgiou et al. (2019). In addition, four EBs have already shown trends of maximum brightness modulation (Papageorgiou et al. 2018), and thus need further monitoring. These systems offer opportunities for further research to clarify the nature of ETVs and possibly detect tertiary companions; this can be accomplished with further light curve analysis, spectroscopy, astrometry, or direct high-resolution imaging. Extension of the eclipse time data sets via new photometric observations will give the opportunity to apply the analytical formula of Irwin (1959) that takes into account the eccentricity of the system. As our main goal is to search for EBs 
Table 3. Period change rate $\frac{d P_{\text {bin }}}{d E}$ for 63 CSS EBs with parabolic ETV variation. Symbols '-' and ' + ' denote the lower and upper bounds, respectively, of the $1 \sigma$ parameter error derived from the MC distributions.

\begin{tabular}{|c|c|c|c|}
\hline Name & $\begin{array}{c}\frac{d P_{\text {bin }}}{d E} \\
\left(\text { days per cycle } \times 10^{-10}\right)\end{array}$ & $\begin{array}{c}\left(\frac{d P_{\text {bin }}}{d E}\right)_{\text {err }} \\
\text { (days per cycle } \times \\
\left.10^{-10}[-,+]\right)\end{array}$ & $\triangle \mathrm{BIC}$ \\
\hline CSS_J002011.4 + 181918 & 57.57 & $18.83,17.42$ & 5.84 \\
\hline CSS_J002712.1 + 062414 & -7.44 & $0.74,0.78$ & 6.45 \\
\hline CSS_J003703.4 + 213021 & 785.23 & $82.43,79.93$ & 21.06 \\
\hline CSS_J010700.6 + 251138 & -591.65 & $75.98,76.21$ & 12.42 \\
\hline CSS_J012942.5 + 220302 & 9.13 & $0.61,0.60$ & 16.05 \\
\hline CSS_J023303.0 + 105943 & -3.79 & $0.35,0.35$ & 8.26 \\
\hline CSS_J025840.7 + 232939 & -51.02 & $13.74,14.46$ & 21.80 \\
\hline CSS_J030325.0 + 250550 & -16.33 & $0.80,0.87$ & 27.12 \\
\hline CSS_J032042.7 + 035254 & 75.85 & $11.39,11.28$ & 21.96 \\
\hline CSS_J040235.7 + 194327 & 72.09 & $7.17,7.81$ & 17.38 \\
\hline CSS_J043713.9+ 024720 & -5.74 & $1.13,1.21$ & 9.62 \\
\hline CSS_J044900.0 + 084800 & 6.58 & $1.32,1.34$ & 9.65 \\
\hline CSS_J045118.8 + 243511 & -1478.00 & $117.38,124.27$ & 12.71 \\
\hline CSS_J045152.6 + 223940 & -15.08 & $2.56,2.33$ & 11.60 \\
\hline CSS_J050212.9 + 101627 & 3.65 & $0.91,0.92$ & 9.43 \\
\hline CSS_J050313.4 + 190251 & 396.82 & $47.21,49.24$ & 14.34 \\
\hline CSS_J050423.2 + 104435 & -1410.16 & $325.06,331.02$ & 7.05 \\
\hline CSS_J050746.1 + 110404 & -322.39 & $18.76,18.90$ & 13.58 \\
\hline CSS_J050804.5 + 073951 & 139.06 & $20.90,19.64$ & 9.43 \\
\hline CSS_J050836.9 + 041255 & 34.75 & $4.14,4.02$ & 16.68 \\
\hline CSS_J070351.6 + 380310 & 181.08 & $6.97,6.88$ & 8.83 \\
\hline CSS_J074403.2 + 465457 & -50.70 & $4.30,4.65$ & 13.19 \\
\hline CSS_J075045.4 + 365022 & 1016.27 & $25.72,23.17$ & 19.03 \\
\hline CSS_J075146.5 + 183257 & -6.26 & $2.15,2.49$ & 11.44 \\
\hline CSS_J075619.6 + 161112 & -72.08 & $14.05,12.67$ & 10.77 \\
\hline CSS_J080105.1 + 103830 & 17.98 & $4.89,4.93$ & 7.87 \\
\hline CSS_J080459.9 + 181851 & 83.13 & $10.67,10.91$ & 15.81 \\
\hline CSS_J082613.8 + 232505 & 51.65 & $4.74,4.86$ & 25.27 \\
\hline CSS_J083522.0 + 074346 & 10.07 & $1.20,1.42$ & 8.11 \\
\hline CSS_J083522.9 + 111829 & -31.88 & $5.34,5.85$ & 12.05 \\
\hline CSS_J083614.1 + 340449 & 468.72 & $61.71,59.16$ & 11.03 \\
\hline CSS_J083709.5 + 241619 & -7.53 & $0.41,0.42$ & 30.70 \\
\hline CSS_J083911.3-022232 & -5.69 & $1.32,1.38$ & 12.08 \\
\hline CSS_J083956.4 + 125534 & 21.43 & $1.49,1.60$ & 9.74 \\
\hline CSS_J085315.4 + 260653 & -10.71 & $1.17,1.26$ & 12.10 \\
\hline CSS_J085530.2 + 250113 & -2.37 & $0.29,0.30$ & 14.08 \\
\hline CSS_J090058.4-021740 & -501.75 & $115.63,120.62$ & 7.33 \\
\hline CSS_J090224.5 + 030715 & -13.67 & $2.64,2.83$ & 13.83 \\
\hline CSS_J090932.6-013538 & -341.26 & $8.75,8.44$ & 29.77 \\
\hline CSS_J091636.7 + 032523 & 17.51 & $2.00,1.90$ & 5.09 \\
\hline CSS_J095937.4 + 091330 & 26.02 & $4.24,4.85$ & 6.32 \\
\hline CSS_J102154.2 + 262840 & 43.55 & $14.75,14.14$ & 5.49 \\
\hline CSS_J105116.4 + 401318 & 8.87 & $1.25,1.15$ & 11.49 \\
\hline CSS_J113435.5 + 042400 & -7.43 & $1.69,1.53$ & 13.27 \\
\hline CSS_J113923.2 + 181058 & 2.02 & $0.47,0.47$ & 5.88 \\
\hline CSS_J120113.8+ 311142 & -19.66 & $4.71,4.40$ & 7.59 \\
\hline CSS_J123432.8-041723 & 2.73 & $0.48,0.46$ & 9.90 \\
\hline CSS_J145611.3 + 284937 & 12.29 & $2.23,2.60$ & 11.28 \\
\hline CSS_J151612.6 + 074630 & -11.47 & $3.52,3.66$ & 10.24 \\
\hline CSS_J152231.8+ 085946 & 19.22 & $1.80,1.79$ & 18.07 \\
\hline CSS_J152246.7 + 122800 & 128.40 & $10.42,10.55$ & 14.42 \\
\hline CSS_J153945.0 + 072046 & 81.43 & $20.36,19.79$ & 14.07 \\
\hline CSS_J160927.3 + 320423 & 283.37 & $28.13,31.65$ & 7.90 \\
\hline CSS_J160957.3 + 161540 & -75.85 & $14.67,14.12$ & 18.38 \\
\hline CSS_J165302.2 + 324640 & -988.60 & $105.43,101.24$ & 10.21 \\
\hline CSS_J165843.3 + 314517 & 9.12 & $1.56,1.48$ & 7.08 \\
\hline CSS_J211621.8-044337 & 8.25 & $0.69,0.72$ & 11.31 \\
\hline CSS_J211655.7 + 010517 & 52.25 & $6.05,6.42$ & 23.38 \\
\hline CSS_J214750.5 + 001527 & 387.14 & $50.99,44.38$ & 6.41 \\
\hline CSS_J215811.0+002246 & 7.05 & $0.64,0.66$ & 18.08 \\
\hline
\end{tabular}


Table 3 - continued

\begin{tabular}{lccr}
\hline Name & $\frac{d P_{\text {bin }}}{d E}$ & $\left(\frac{d P_{\text {bin }}}{d E}\right)_{\text {err }}$ \\
& $($ days per cycle $\times$ \\
$\left.10^{-10}[-,+]\right)$ & $\Delta$ BIC \\
\hline CSS_J2222220.0+210144 & -250.94 & $57.16,56.36$ & \\
CSS_J230624.5 + 350053 & -12.70 & $3.05,3.11$ & 7.79 \\
CSS_J232646.8 +313030 & 53.87 & $5.28,5.44$ & 11.23 \\
\hline
\end{tabular}
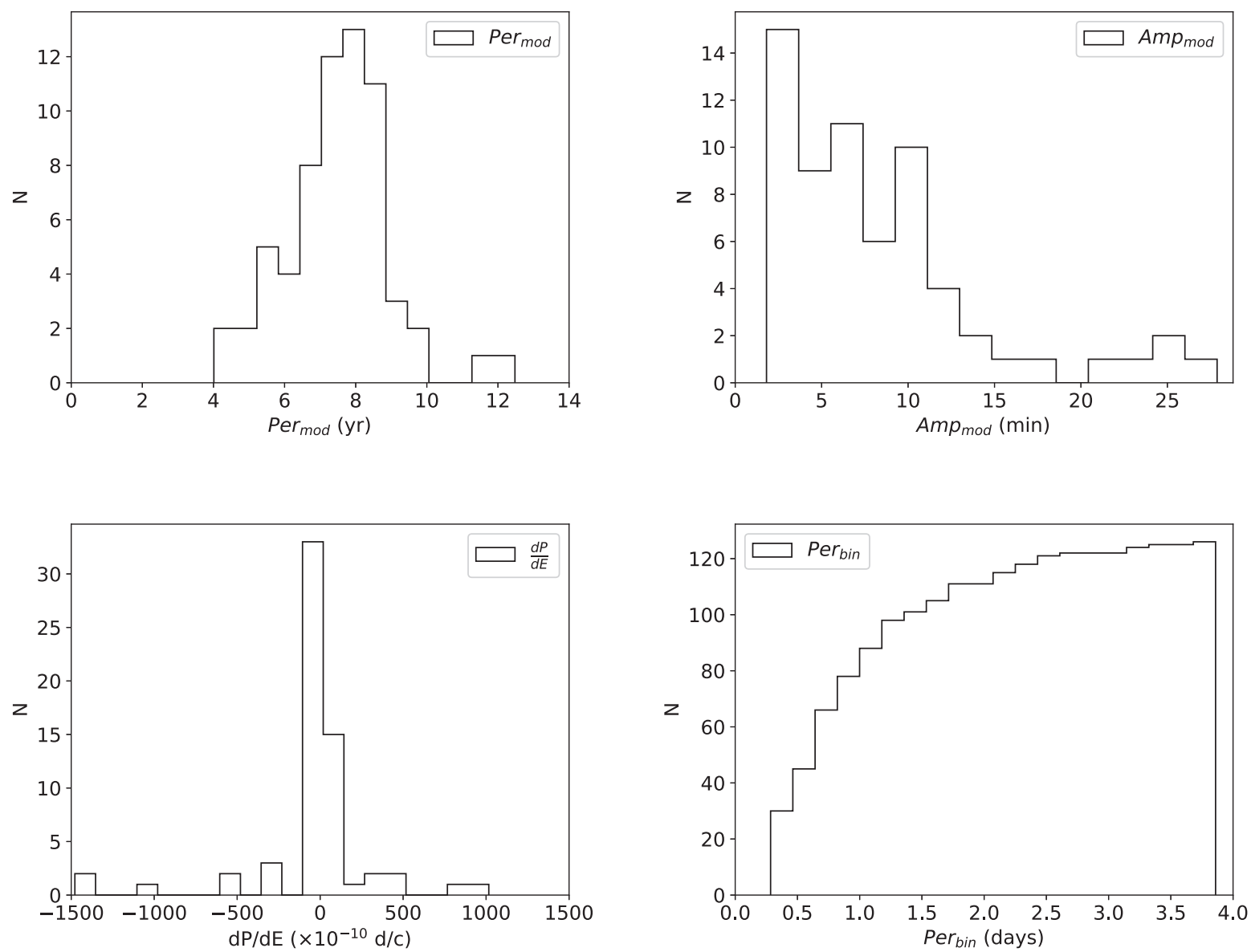

Figure 5. Distributions of the period modulation $P e r_{\text {mod }}$ (top left), amplitude $A m p_{\text {mod }}$ (top right), period change rate $\frac{d P_{\text {bin }}}{d E}$ (bottom left), and cumulative distribution of the binary period $P_{\text {bin }}$ (bottom right) of CSS EBs.

with considerable ETVs, we used a simple sinusoidal fitting instead of looking for a more complicated solution in the $O-C$ analysis. In the near future we will focus on individual more interesting and promising EBs in which the number of data points ToM from this analysis is large enough. These will be used together with a compilation of ToM from archival data and new that will be determined from cross-matching with other surveys, for a more sophisticated $O-C$ analysis and search for a global solution in the parameter space.

Our results are consistent with the finding that the frequency of EBs with potential tertiary component increases as the period of the binary decreases, as previously found using OGLE-IV and MOA data. The lack of sub-stellar detections is explained by our lower detection limit $\left(A m p_{\text {mod }} \sim 2 \mathrm{~min}\right)$, but stellar companions of comparable mass to the mass of the EB components are detected Tokovinin (2008).

The distribution of the ETVs periods is biased due to the observing time span and the selection criteria. The majority of the EBs in the final sample have periods shorter than $\sim 2 \mathrm{~d}$ (Fig. 5, bottom right), as does the majority of the initial sample of 4683 EBs. In order to discriminate between cyclical ETVs produced by a third body and those produced by a magnetic dynamo effect (e.g. the Applegate mechanism), we would need to observe the constancy of the observed cyclical periods of the ETVs. Thus, long-term observations of the candidate systems with cyclical ETVs can provide a useful method to discriminate between the presence of a third body and other non-circumbinary effects. This would be strongly desirable, as multiplicity and mass transfer/loss are the key pro- 


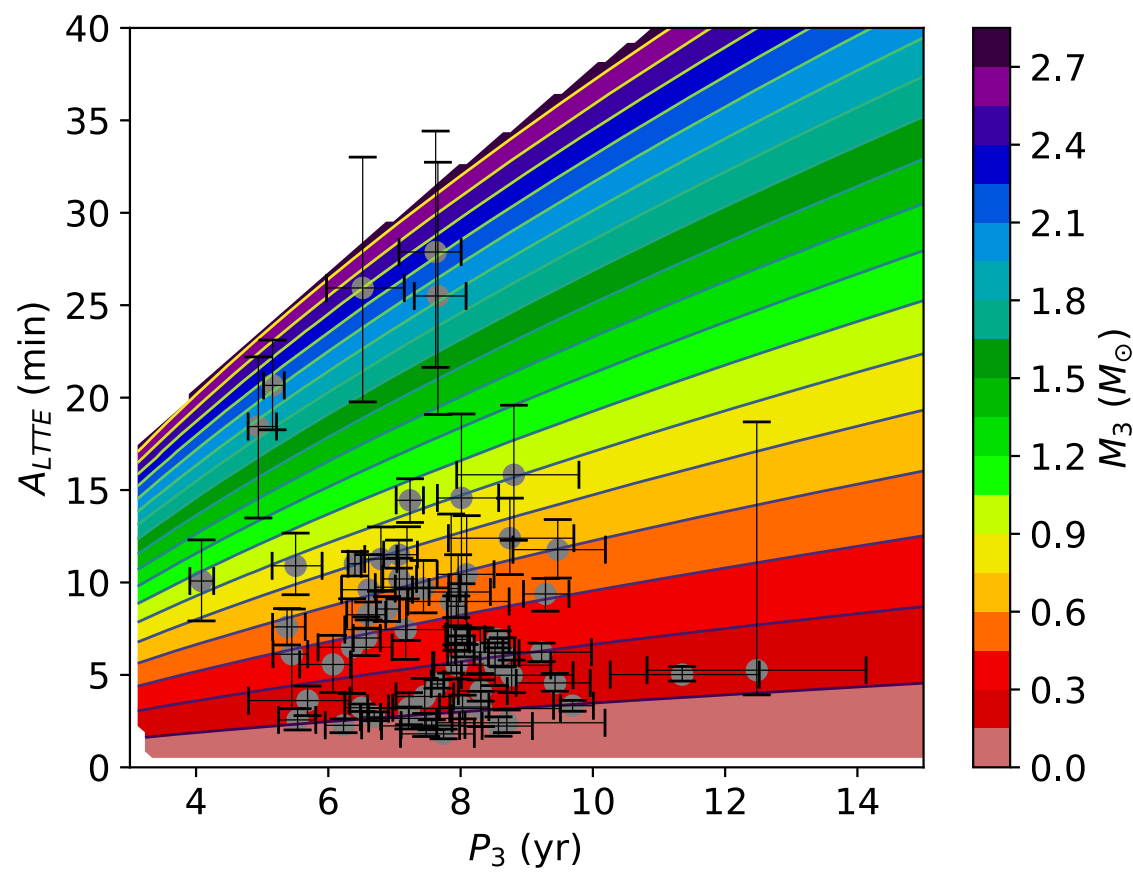

Figure 6. Calculated minimum masses of the tertiary companion $\left(M_{3}\right)$ for 63 triple candidates. The contours for the tertiary mass were generated by using 10000 random pairs of $A_{\mathrm{LTTE}}$ and $P_{3}$ values, assuming a $2 \mathrm{M}_{\odot}$ EB system.

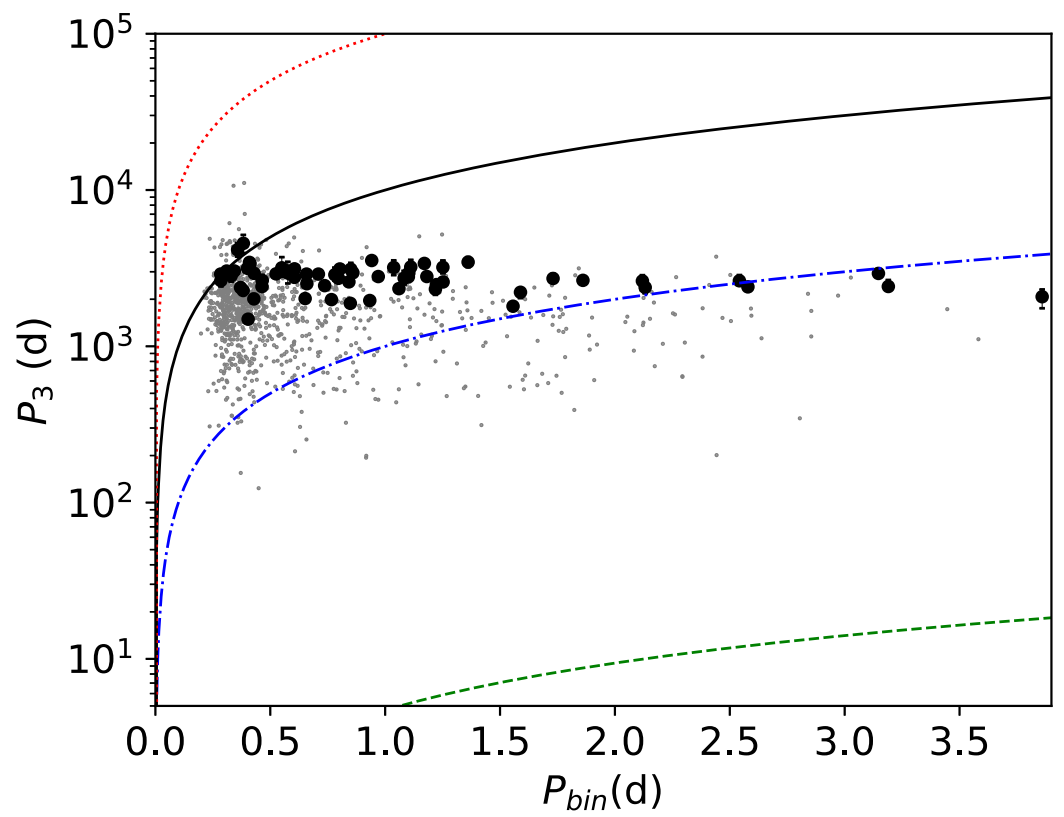

Figure 7. Binary period $\left(P_{\text {bin }}\right)$ versus period of the ETVs modulation $\left(P_{3}\right)$ for 63 triple candidates from our study (large circles). The 992 potential triple candidates from OGLE-IV (Hajdu et al. 2019) are also shown, for comparison (grey dots). Overplotted are loci corresponding to log $\left(P_{3} / P_{\text {bin }}\right)=3($ dash-dotted blue line), 4 (solid line), and 5 (dotted red line). The dashed green line represents the limit for stable orbits of the third body.

cesses that drive the evolution of EBs towards contact and eventual mergers.

\section{ACKNOWLEDGEMENTS}

AP and MC gratefully acknowledge the support provided by FONDECYT through grants \#3160782 and \#1171273. Additional support for this project is provided by the Ministry for the Economy,
Development, and Tourism's Millennium Science Initiative through grant IC 120009, awarded to the Millennium Institute of Astrophysics (MAS); and by Proyecto Basal AFB-170002. We would like to thank the anonymous reviewer for the suggestions that helped to improve the paper.

This work made use of data products from the CSS survey. The CSS survey is funded by the National Aeronautics and Space Administration under Grant No. NNG05GF22G issued through 


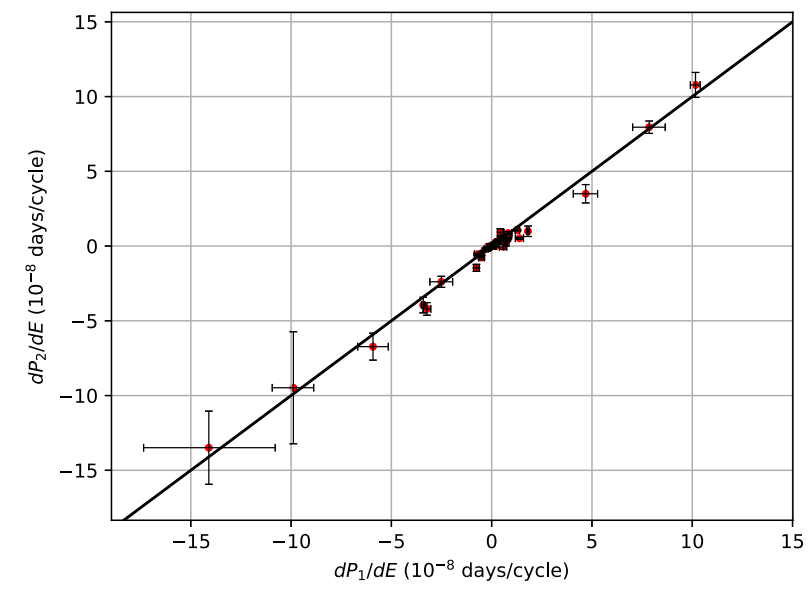

Figure 8. Comparison between the period change rate derived from the ETV fitting $\left(\frac{d P_{1}}{d E}\right)$ and the period change rate derived from the LCs harmonic fit $\left(\frac{d P_{2}}{d E}\right)$ for 58 CSS EBs. The line denotes $x=y$.

the Science Mission Directorate Near-Earth Objects Observations Program. The CRTS survey is supported by the US National Science Foundation under grants AST-0909182, AST-1313422, AST-1413600, and AST-1518308.

\section{DATA AVAILABILITY}

The data underlying this article are available in the article and in its online supplementary material.

\section{REFERENCES}

Andrych K. D., Tvardovskyi D. E., Chinarova L. L., Andronov I. L., 2020, Contrib. Astron. Obs. Skaln. Pleso, 50, 557

Applegate J. H., 1992, ApJ, 385, 621

Bertin E., Arnouts S., 1996, A\&AS, 117, 393

Bin Z., Sheng-Bang Q., Nian-Ping L., Qi-Jun Z., Li-Ying Z., Ai-Jun D., Lin-Qiao J., 2019, New Astron., 70, 1

Borkovits T., Érdi B., Forgács-Dajka E., Kovács T., 2003, A\&A, 398, 1091

Borkovits T., Csizmadia S., Forgács-Dajka E., Hegedüs T., 2011, A\&A, 528, A53

Borkovits T., Rappaport S., Hajdu T., Sztakovics J., 2015, MNRAS, 448, 946

Borkovits T., Hajdu T., Sztakovics J., Rappaport S., Levine A., Bíró I. B., Klagyivik P., 2016, MNRAS, 455, 4136

Borkovits T. et al., 2019a, MNRAS, 483, 1934

Borkovits T., Sperauskas J., Tokovinin A., Latham D. W., Csányi I., Hajdu T., Molnár L., 2019b, MNRAS, 487, 4631

Borkovits T. et al., 2020, MNRAS, 496, 4624

Brown W. R., Kilic M., Gianninas A., 2017, ApJ, 839, 23

Conroy K. E., Prša A., Stassun K. G., Orosz J. A., Fabrycky D. C., Welsh W. F., 2014, AJ, 147, 45

Conroy K. E., Prša A., Horvat M., Stassun K. G., 2018, ApJ, 854, 163

Drake A. J. et al., 2013, ApJ, 763, 32

Drake A. J. et al., 2014, ApJS, 213, 9

Eggleton P. P., Kisseleva-Eggleton L., 2006, Ap\&SS, 304, 75

Erdem A., Öztürk O., 2014, MNRAS, 441, 1166

Foreman-Mackey D., Hogg D. W., Lang D., Goodman J., 2013, PASP, 125, 306

Gaia Collaboration, 2018, A\&A, 616, A1

Graham M. J., Djorgovski S. G., Drake A. J., Stern D., Mahabal A. A., Glikman E., Larson S., Christensen E., 2017, MNRAS, 470, 4112

Hajdu T., Borkovits T., Forgács-Dajka E., Sztakovics J., Marschalkó G., Benkő J. M., Klagyivik P., Sallai M. J., 2017, MNRAS, 471, 1230
Hajdu T., Borkovits T., Forgács-Dajka E., Sztakovics J., Marschalkó G., Kutrovátz G., 2019, MNRAS, 485, 2562

Hoffman D. I., Harrison T. E., McNamara B. J., Vestrand W. T., Holtzman J. A., Barker T., 2006, AJ, 132, 2260

Ibanoğlu C., Soydugan F., Soydugan E., Dervisoğlu A., 2006, MNRAS, 373, 435

Iglewicz B., Hoaglin David C., 1993, How to Detect and Handle Outliers. ASQC Quality Press, Milwaukee, WI

Irwin J. B., 1959, AJ, 64, 149

Ivezić Ž. et al., 2019, ApJ, 873, 111

Kwee K. K., van Woerden H., 1956, Bull. Astron. Inst. Netherlands, 12, 327

Lanza A. F., Rodonò M., 2004, Astron. Nachr., 325, 393

Levenberg K., 1944, Q. Appl. Math., 2, 164

Li M. C. A. et al., 2018, MNRAS, 480, 4557

Lohr M. E., Norton A. J., Payne S. G., West R. G., Wheatley P. J., 2015, A\&A, 578, A136

Marquardt D. W., 1963, J. Soc. Ind. Appl. Math., 11, 431

Mikulášek Z., 2015, A\&A, 584, A8

Mowlavi N. et al., 2017, A\&A, 606, A92

Navarrete F. H., Schleicher D. R. G., Zamponi Fuentealba J., Völschow M., 2018, A\&A, 615, A81

Nelder J. A., Mead R., 1965, Comput. J., 7, 308

Newville M., Stensitzki T., Allen D. B., Rawlik M., Ingargiola A., Nelson A., 2016, Lmfit: Non-Linear Least-Square Minimization and Curve-Fitting for Python, (ascl:1606.014)

Papageorgiou A., Catelan M., Christopoulou P.-E., Drake A. J., Djorgovski S. G., 2018, ApJS, 238, 4

Papageorgiou A., Catelan M., Christopoulou P.-E., Drake A. J., Djorgovski S. G., 2019, ApJS, 242, 6

Pilecki B., Fabrycky D., Poleski R., 2007, MNRAS, 378, 757

Pojmanski G., 1997, AcA, 47, 467

Pojmanski G., Pilecki B., Szczygiel D., 2005, AcA, 55, 275

Pribulla T., Chochol D., Milano L., Errico L., Vittone A. A., Barone F., Parimucha Š., 2000, A\&A, 362, 169

Pribulla T. et al., 2012, Astron. Nachr., 333, 754

Prša A., Guinan E. F., Devinney E. J., DeGeorge M., Bradstreet D. H., Giammarco J. M., Alcock C. R., Engle S. G., 2008, ApJ, 687, 542

Prša A. et al., 2016, ApJS, 227, 29

Rappaport S., Deck K., Levine A., Borkovits T., Carter J., El Mellah I., Sanchis-Ojeda R., Kalomeni B., 2013, ApJ, 768, 33

Schwarz G., 1978, Ann. Stat., 6, 461

Soszyński I. et al., 2016, AcA, 66, 405

Sterken C., 2005, in Sterken C., ed., ASP Conf. Ser. Vol. 335, The Light-Time Effect in Astrophysics: Causes and Cures of the O-C Diagram. Astron. Soc. Pac., San Francisco

Sumi T. et al., 2013, ApJ, 778, 150

Tokovinin A., 2008, MNRAS, 389, 925

Völschow M., Schleicher D. R. G., Perdelwitz V., Banerjee R., 2016, A\&A, 587, A34

Wolf M. et al., 2016, A\&A, 587, A82

Zasche P., Wolf M., Vraštil J., Liška J., Skarka M., Zejda M., 2014, A\&A, 572, A71

Zhang B., Qian S.-B., Liao W.-P., Zhu L.-Y., Dong A.-J., Zhi Q.-J., 2018, RAA, 18, 116

\section{SUPPORTING INFORMATION}

Supplementary data are available at MNRAS online.

\section{Table1_Supplementry.ascii}

Please note: Oxford University Press is not responsible for the content or functionality of any supporting materials supplied by the authors. Any queries (other than missing material) should be directed to the corresponding author for the article. 


\section{APPENDIX A: ETV DIAGRAMS OF CSS EBS}

Figs A1-A10 provide ETV diagrams for the 126 EBs with evidence of period variations, based on our analysis of CSS data.
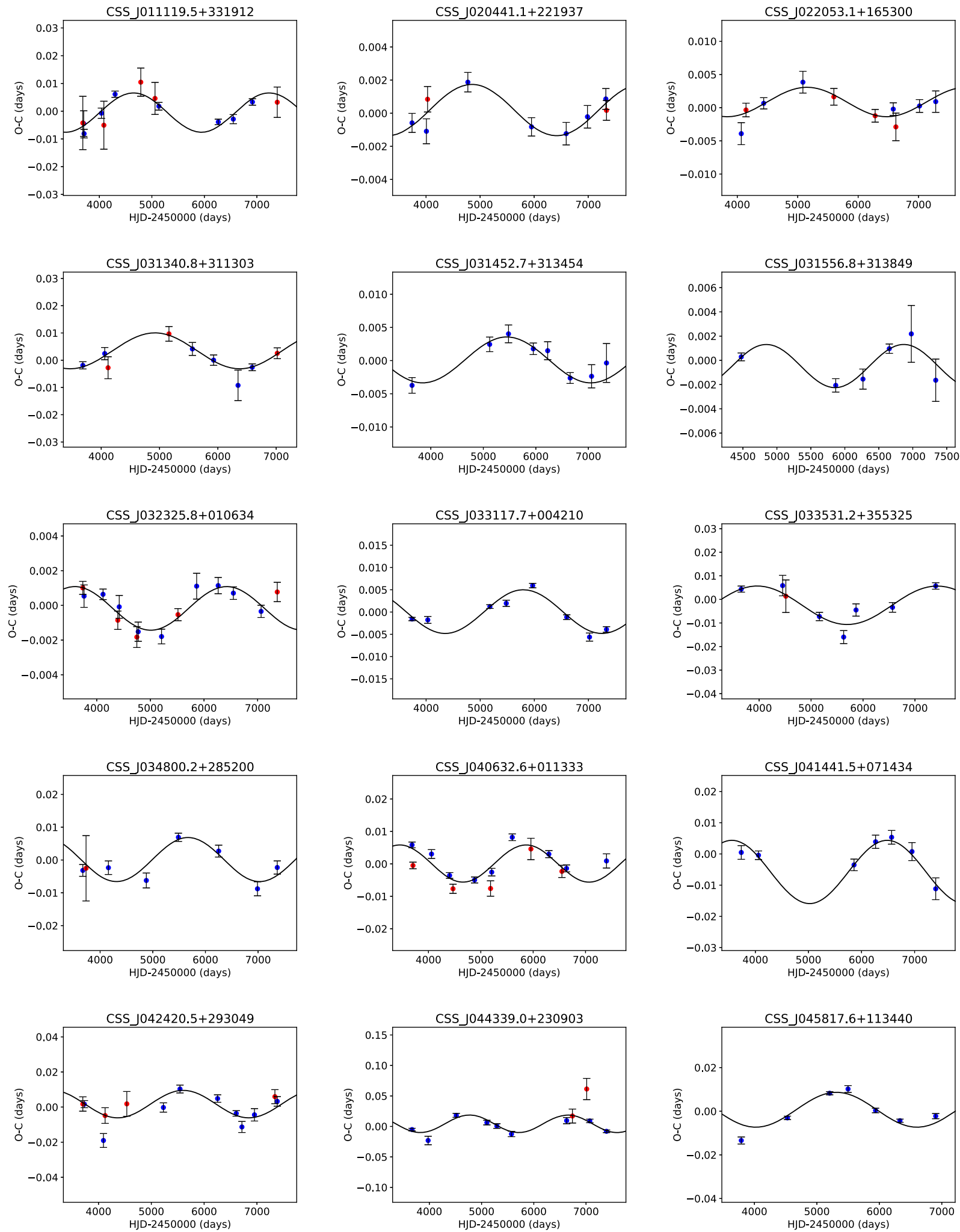

Figure A1. ETV curves of 15 EBs from CSS with evidence of sinusoidal period variation. 

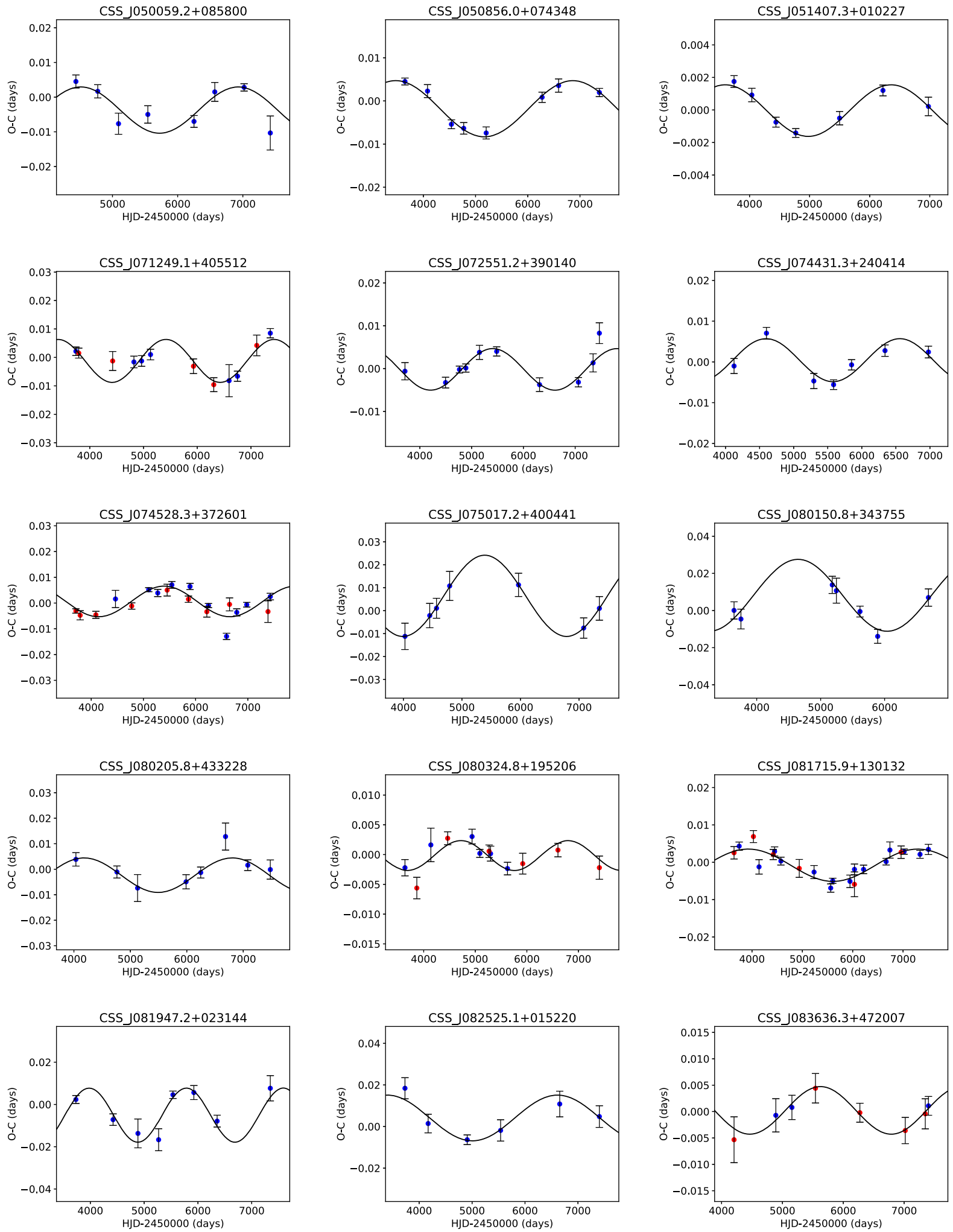

Figure A2. As in Fig. A1, but for an additional 15 systems. 

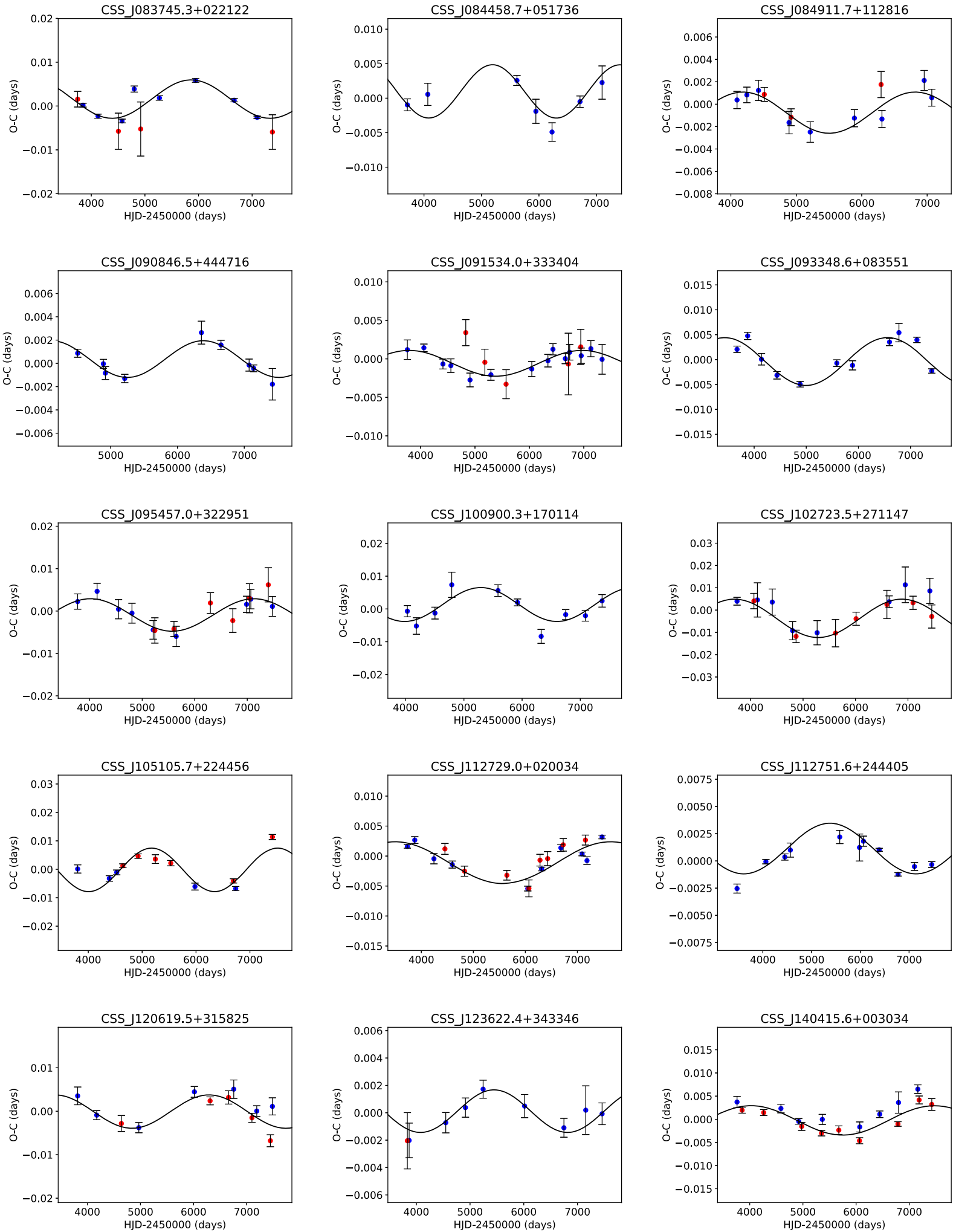

Figure A3. As in Fig. A1, but for an additional 15 systems. 

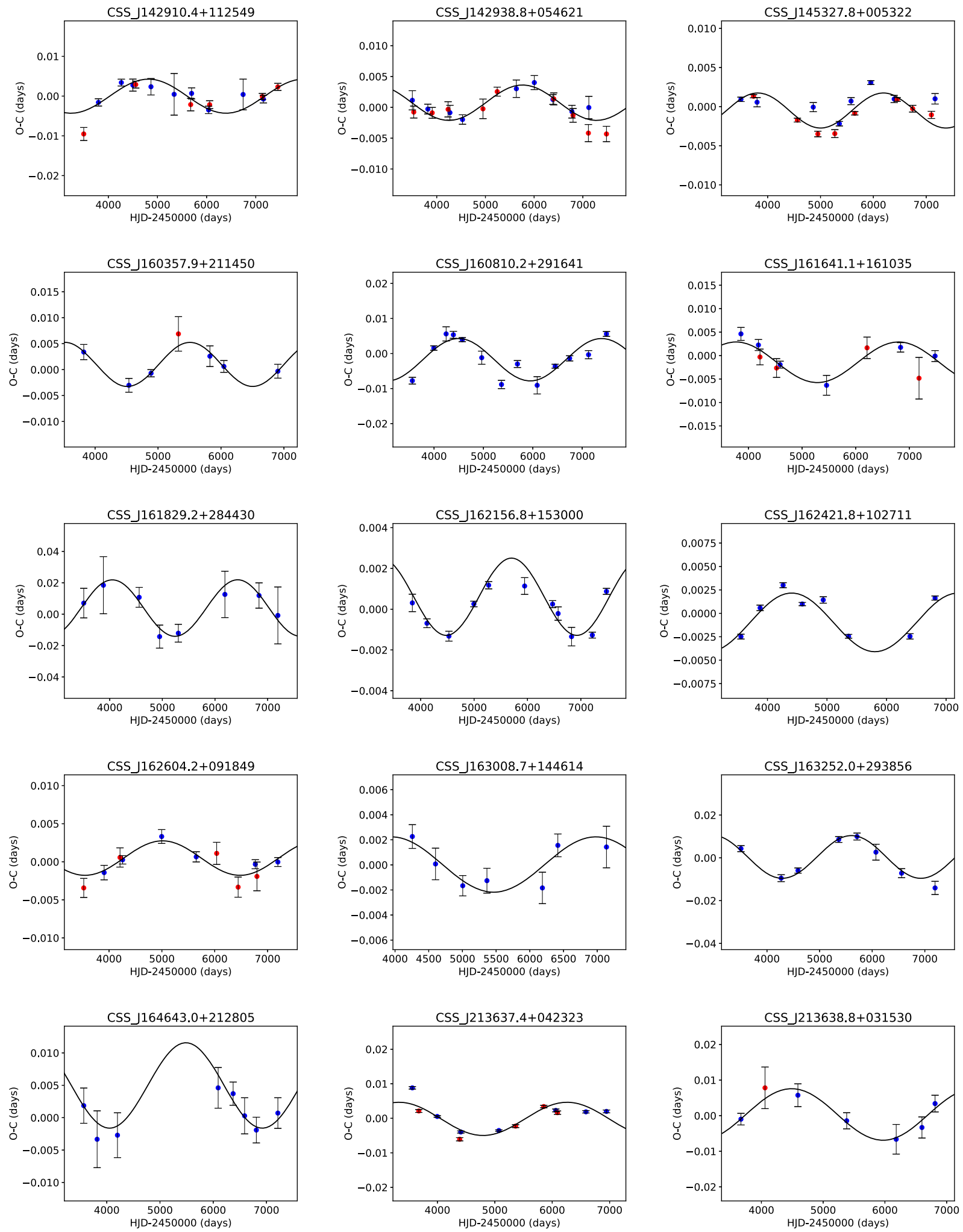

Figure A4. As in Fig. A1, but for an additional 15 systems. 

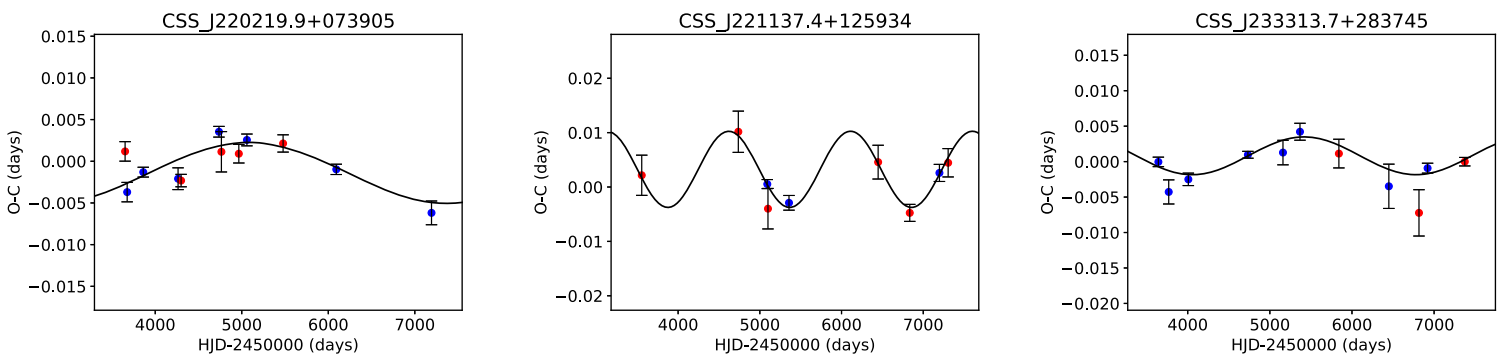

Figure A5. As in Fig. A1, but for an additional three systems.
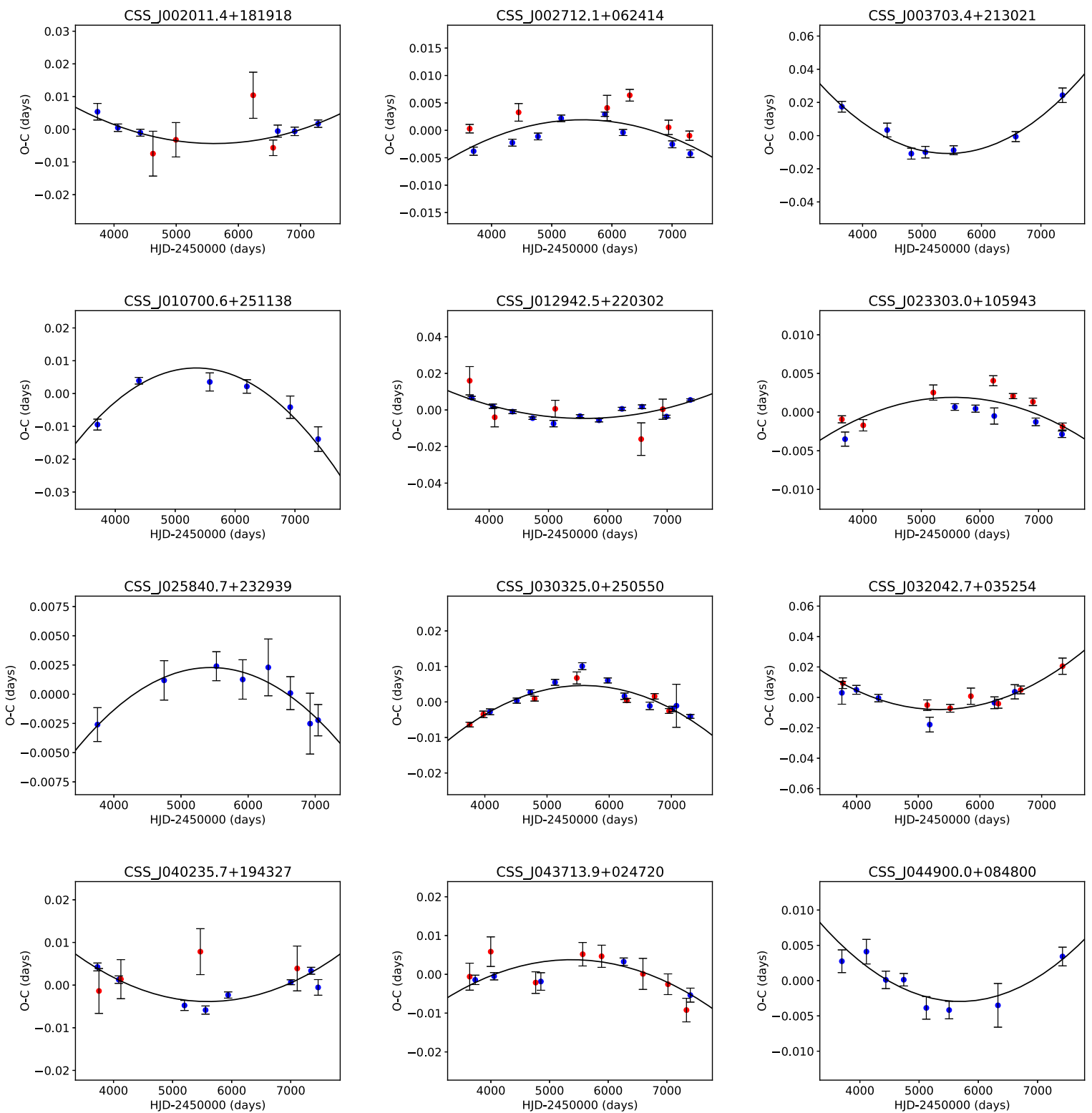

Figure A6. ETV curves of 12 EBs from CSS with evidence of parabolic period variation. 

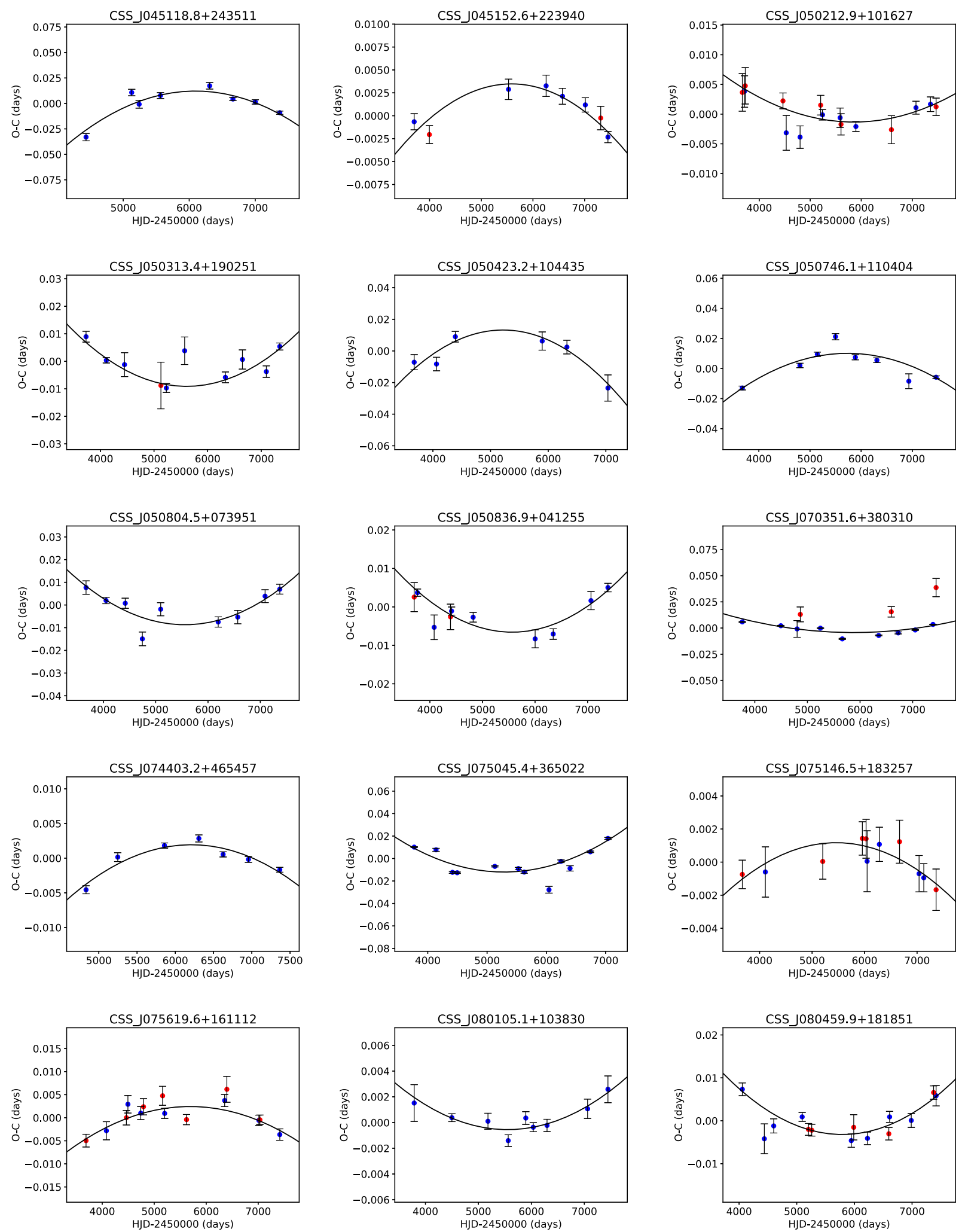

Figure A7. As in Fig. A6, but for an additional 15 systems. 

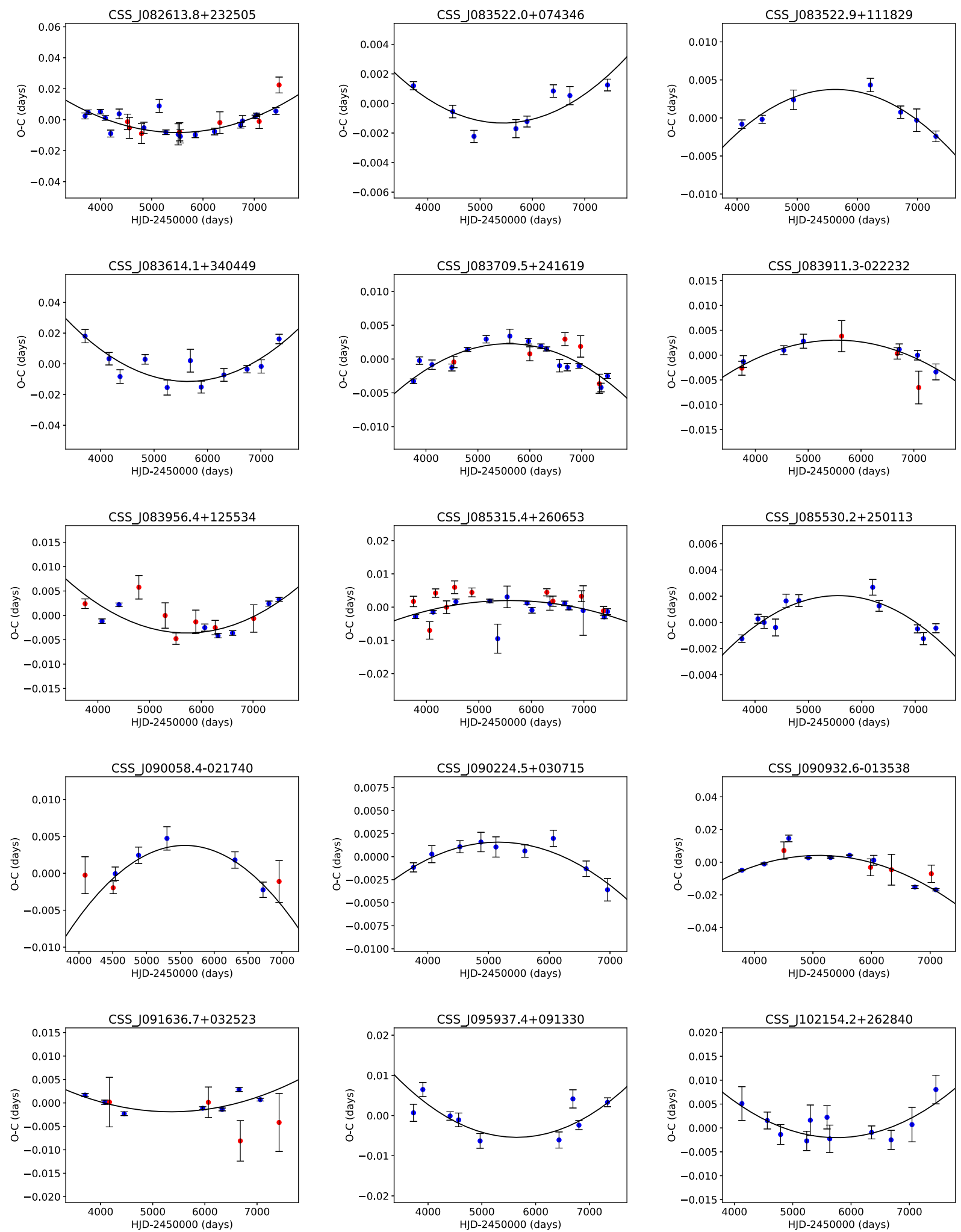

Figure A8. As in Fig. A6, but for an additional 15 systems. 

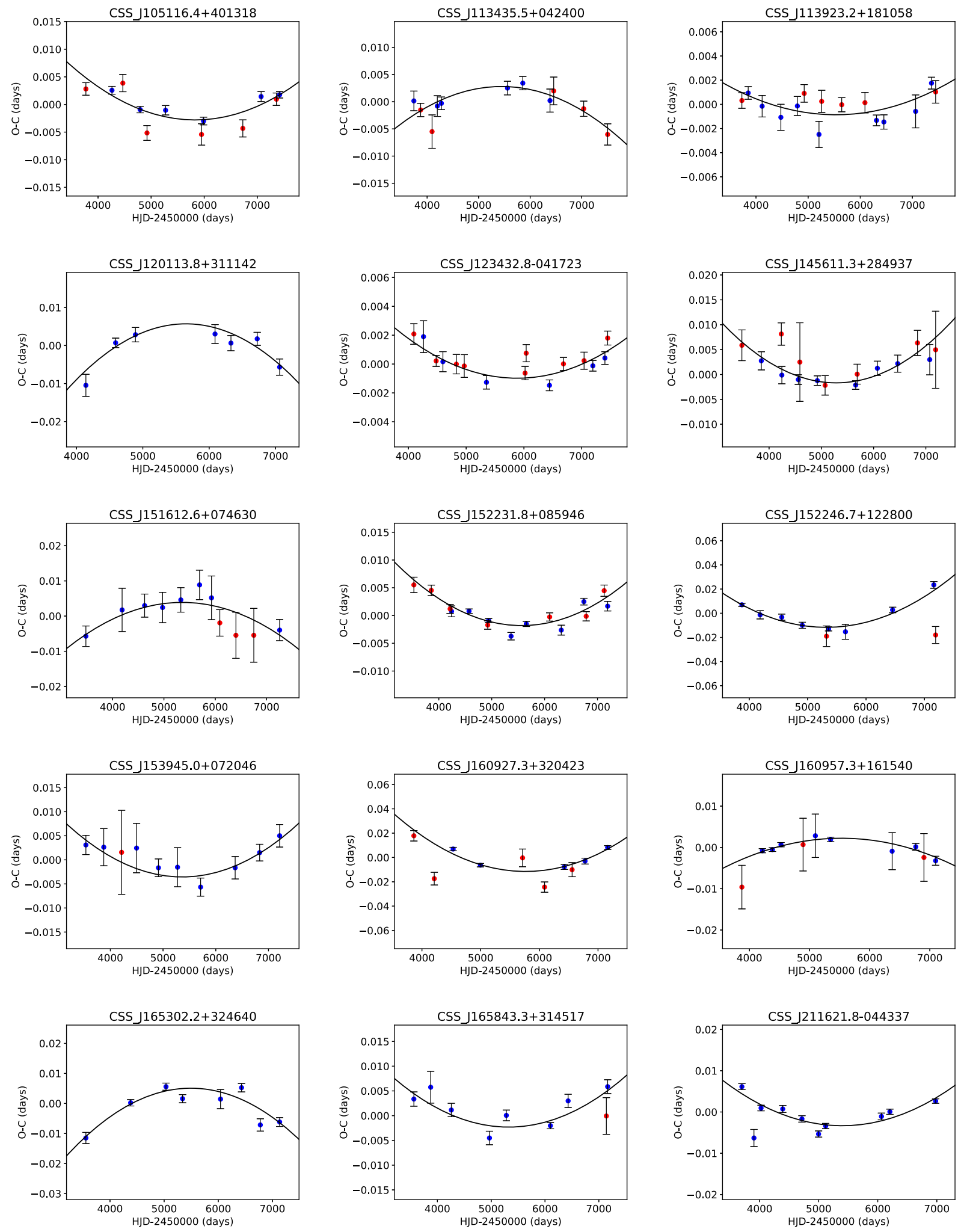

Figure A9. As in Fig. A6, but for an additional 15 systems. 
CSS J211655.7+010517

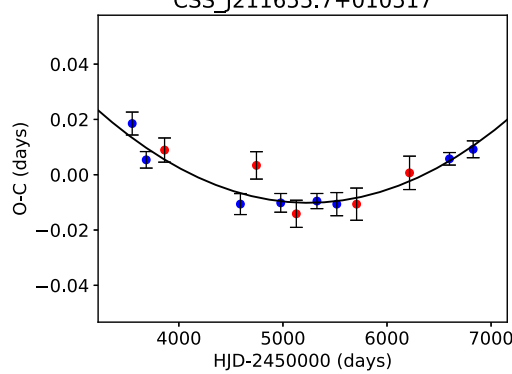

CSS $J 222220.0+210144$

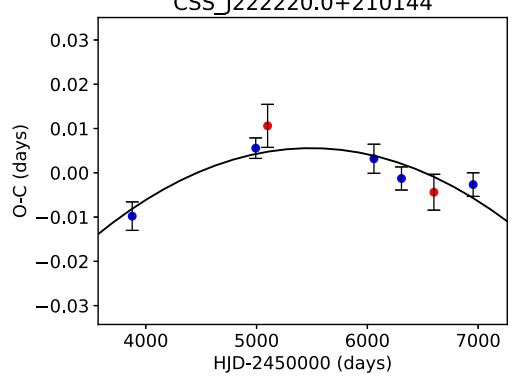

CSS $J 214750.5+001527$

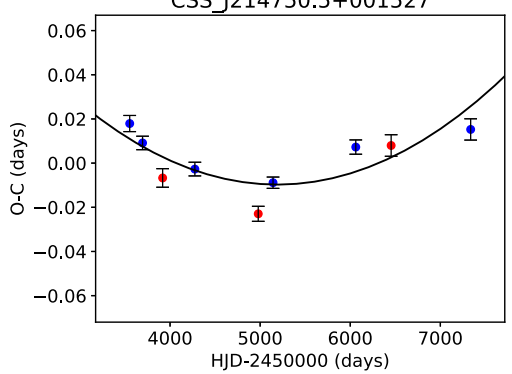

CSS $\_230624.5+350053$

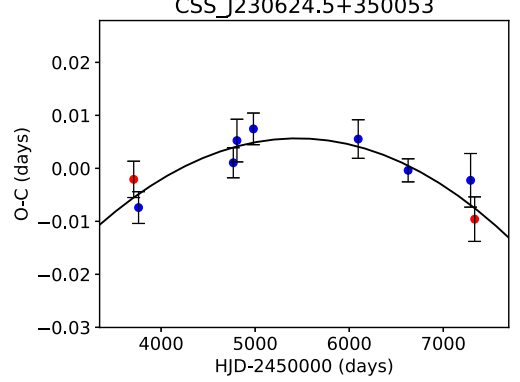

CSS J215811.0+002246

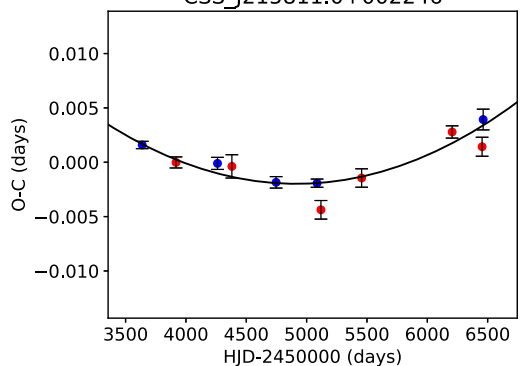

CSS $\_232646.8+313030$

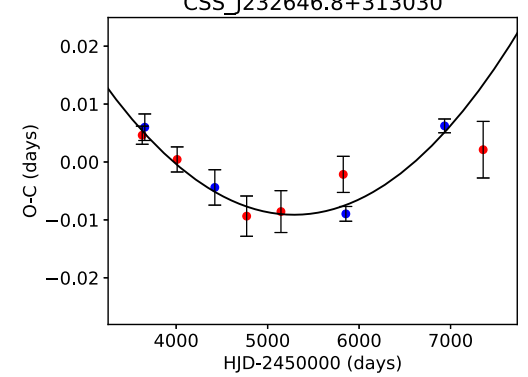

Figure A10. As in Fig. A6, but for an additional six systems.

This paper has been typeset from a $\mathrm{T}_{\mathrm{E}} \mathrm{X} / \mathrm{LTT}_{\mathrm{E}} \mathrm{X}$ file prepared by the author. 